\title{
Gene Expression Profiling During Asexual Development of the Late Blight Pathogen Phytophthora infestans Reveals a Highly Dynamic Transcriptome
}

\author{
Howard S. Judelson, ${ }^{1}$ Audrey M. V. Ah-Fong, ${ }^{1}$ George Aux, ${ }^{2}$ Anna O. Avrova, ${ }^{3}$ Catherine Bruce, ${ }^{4}$ \\ Cahid Cakir, ${ }^{5}$ Luis da Cunha, ${ }^{5}$ Laura Grenville-Briggs, ${ }^{4}$ Maita Latijnhouwers, ${ }^{6}$ Wilco Ligterink, ${ }^{6}$ \\ Harold J. G. Meijer, ${ }^{6}$ Samuel Roberts, ${ }^{1}$ Carrie S. Thurber, ${ }^{1}$ Stephen C. Whisson, ${ }^{3}$ Paul R. J. Birch, ${ }^{3}$ \\ Francine Govers, ${ }^{6}$ Sophien Kamoun, ${ }^{5}$ Pieter van West, ${ }^{4}$ and John Windass ${ }^{7}$ \\ ${ }^{1}$ Department of Plant Pathology and Microbiology, University of California, Riverside 92521, U.S.A.; ${ }^{2}$ Syngenta Biotechnology, \\ Research Triangle Park, NC 27709, U.S.A.; ${ }^{3}$ Plant Pathology Programme, Scottish Crop Research Institute, Invergowrie, \\ Dundee DD2 5DA, U.K.; ${ }^{4}$ College of Life Sciences and Medicine, Institute of Medical Sciences, University of Aberdeen, \\ Foresterhill, Aberdeen AB25 2ZD, U.K.; ${ }^{5}$ Department of Plant Pathology, The Ohio State University, Wooster 44691, U.S.A.; \\ ${ }^{6}$ Laboratory of Phytopathology, Wageningen University, NL-6709 PD Wageningen, The Netherlands; ${ }^{7}$ Syngenta Limited, \\ Jealott's Hill International Research Station, Bracknell, Berks RG42 6EY, U.K.
}

Submitted 10 October 2007. Accepted 19 November 2007.

Much of the pathogenic success of Phytophthora infestans, the potato and tomato late blight agent, relies on its ability to generate from mycelia large amounts of sporangia, which release zoospores that encyst and form infection structures. To better understand these stages, Affymetrix GeneChips based on 15,650 unigenes were designed and used to profile the life cycle. Approximately half of $\boldsymbol{P}$. infestans genes were found to exhibit significant differential expression between developmental transitions, with approximately $1 / 10$ being stage-specific and most changes occurring during zoosporogenesis. Quantitative reverse-transcription polymerase chain reaction assays confirmed the robustness of the array results and showed that similar patterns of differential expression were obtained regardless of whether hyphae were from laboratory media or infected tomato. Differentially expressed genes encode potential cellular regulators, especially protein kinases; metabolic enzymes such as those involved in glycolysis, gluconeogenesis, or the biosynthesis of amino acids or lipids; regulators of DNA synthesis; structural proteins, including predicted flagellar proteins; and pathogenicity factors, including cell-wall-degrading enzymes, RXLR effector proteins, and enzymes protecting against plant defense responses. Curiously, some stage-specific transcripts do not appear to encode functional proteins. These findings reveal many new aspects of oomycete biology, as well as potential targets for crop protection chemicals.

Additional keywords: appressorium, microarray, sporulation.

Integral to the fitness of most phytopathogenic microbes are their abilities to transition between stages dedicated for func-

Current address for S. Kamoun: The Sainsbury Laboratory, John Innes Center, Norwich NR4 7UH, U.K.

Corresponding author: H. Judelson; E-mail: howard.judelson@ucr.edu

Microarray data are deposited in the National Center for Biotechnology Information Gene Expression Omnibus as series GSE9623.

* The $\boldsymbol{e}$-Xtra logo stands for "electronic extra" and indicates that two supplemental tables are available online. tions such as dispersal or host invasion. This is especially true for species that persist poorly in the environment as saprophytes or for which host recognition or colonization involves specialized cellular structures. Such features characterize Phytophthora infestans, which causes the economically important late blight diseases of potato and tomato (Smart and Fry 2001). Phytophthora spp. and their oomycete relatives, such as those in the Pythium and downy mildew groups, display fungus-like patterns of growth yet lack strong taxonomic affinity with true fungi. Instead, these species reside within the kingdom Stramenopila, which also includes brown algae and diatoms (Baldauf et al. 2000). Although many oomycetes have been well studied using classical microbiological approaches, much remains to be learned of the molecular features of their life cycles.

Most oomycetes exhibit both sexual and asexual cycles, but the latter is most important in late blight. Growth of $P$. infestans in nature entails the extension of branched and aseptate filamentous hyphae within a host. Upon the termini of specialized hyphae, multinucleate asexual sporangia develop which are spread by wind or water to new potential sites of infection (Judelson and Blanco 2005). Although sporangia can extend germ tubes directly, common to the cool and moist conditions conducive to epidemics is the formation of the uninucleate and biflagellated zoospores. This involves a process referred to as "cleavage," in which vesicles and other sporangial components reorganize into zoospores (Hardham 2001). Zoospores are chemotactic and stimulated by host exudates to shed their flagella, form a cyst wall, and release adhesive glycoproteins that facilitate binding to plant surfaces, all within about a minute. After approximately $2 \mathrm{~h}$, each cyst extends a germ tube that subsequently swells to form an appressorium upon hydrophobic surfaces, such as the plant cuticle. Inhibitor studies suggested that sporangia contain the factors needed for zoosporogenesis and cyst formation, whereas de novo transcription and translation are required for cyst germination and appressorium formation (Clark et al. 1978; Penington et al. 1989). Hydrolytic enzymes released from the appressorium and physical pressure combine to enable the plant wall to be breached by a penetration peg, which develops into hyphae that ramify through the plant. $P$. infestans also can enter certain tissues, such as potato tubers, through natural openings or wounds without passing 
through a spore stage. However, epidemics rely on the production of spores. During favorable weather, a complete asexual cycle takes as little as 5 days and, consequently, many rounds of sporangia dispersal, zoospore release, and infection can occur within a single season. This helps to explain why $P$. infestans is such a devastating pathogen.

$P$. infestans presents a good model for studying the oomycete life cycle. It is easily cultured, large amounts of sporangia can be purified from hyphae, and each developmental stage described above can be induced without a plant host, which is not the case for many oomycetes (Hardham and Hyde 1997). Also, progress has begun to be made toward identifying genes and proteins involved in the different life stages. For example, expressed sequence tags (EST) were generated from many stages of the life cycle, including hyphae, sporangia, cleaving sporangia, swimming zoospores, and germinating zoospore cysts (Randall et al. 2005). Potential participants in development were identified based on protein or RNA expression patterns, and several were shown to play important roles through the application of transformation-based gene silencing methods (Ah Fong and Judelson 2003; Avrova et al. 2003; Blanco and Judelson 2005; Goernhardt et al. 2000; Grenville-Briggs et al. 2005; Judelson and Tani 2007; Kim and Judelson 2003; Latijnhouwers and Govers 2003; Latijnhouwers et al. 2004; Tani et al. 2004).

Despite such advances, an overall picture of the molecular events occurring during the Phytophthora life cycle remains to be developed. In other microbes, the use of microarrays has proved to be a valuable approach for gaining insight into development and pathogenesis (Guldener et al. 2006; Takano et al. 2003; Zahiri et al. 2005). Here, we describe the large-scale profiling of the $P$. infestans transcriptome during asexual development, using Affymetrix GeneChips based on a unigene set developed from EST and partial genomic sequencing. A large fraction of the transcriptome is shown to be differentially expressed during the life cycle, with $>50 \%$ of genes exhibiting significant changes in mRNA abundance during developmental transitions, including 1,843 genes induced $>100$-fold between stages. Differentially expressed genes include many potential regulators of cellular processes, metabolic enzymes, structural proteins, and pathogenicity factors.

\section{RESULTS AND DISCUSSION}

\section{Design and evaluation of the microarray.}

$P$. infestans sequences used to construct the GeneChip were based on a set of 18,256 unigenes. These were assembled from 76,000 EST and genomic sequences of high coding potential detected in a $1 \times$ genome skim (Randall et al. 2005). Stages represented by the EST libraries included those that are the focus of this article; namely, nonsporulating hyphae, sporangia, cleaving sporangia, swimming zoospores, and germinating zoospore cysts. Within the unigene set, 15,926 sequences had EST support whereas 2,330 were derived from genomic reads alone. In total, 15,650 unigenes met Affymetrix criteria for probe design and were included on the array, including 6,803 supported by multiple EST, 6,589 from EST singletons, and 2,258 by ge-

Table 1. Construction and performance of Phytophthora infestans GeneChip

\begin{tabular}{lccc}
\hline & \multicolumn{3}{c}{ Number of unigenes } \\
\cline { 2 - 4 } $\begin{array}{l}\text { Support for } \\
\text { unigene }^{\mathbf{a}}\end{array}$ & $\begin{array}{c}\text { Represented } \\
\text { on array }\end{array}$ & $\begin{array}{c}\text { Absent in 85 of } \\
\text { 89 experiments }\end{array}$ & $\begin{array}{c}\text { Absent in all } \\
\text { 89 experiments }\end{array}$ \\
\hline EST contigs & 6,803 & $394(6 \%)$ & $187(3 \%)$ \\
EST singletons & 6,589 & $1,340(20 \%)$ & $749(11 \%)$ \\
Genomic reads only & 2,258 & $982(43 \%)$ & $655(29 \%)$ \\
Total & 15,650 & $2,716(17 \%)$ & $1,591(10 \%)$ \\
\hline
\end{tabular}

${ }^{\mathrm{a}} \mathrm{EST}=$ expressed sequence tag. nomic reads alone (Table 1). In the rest of this article, these will be referred to as genes even though not all may be protein-coding and some redundancy may exist between unigenes. Their sequences, described here using the "Pi" prefix, are deposited as Platform GPL4523 in the National Center for Biotechnology Information Gene Expression Omnibus (NCBI GEO) archive.

Several approaches appraised the technical quality of the array and analysis pipeline. To evaluate how many genes were detectable, hybridizations were performed using RNA from 89 tissues. These included the developmental stages addressed by this article (germinated and ungerminated asexual spores, plus hyphae), other stages such as oosporogenesis (Prakob and Judelson 2007), and miscellaneous chemical treatments. Of the 15,650 genes, 14,059 appeared to be expressed under at least one condition (i.e., a present flag was called by the Affymetrix MAS algorithm). Hybridization levels for 1,591 were considered insignificant (i.e., yielded absent calls) in all treatments, while 2,716 were absent in $95 \%$ of experiments (Table 1). Approximately 4,000 genes were flagged as present in all 89 conditions. Not surprisingly, genes with the strongest EST support were more likely to be scored as expressed. For example, 97\% of genes supported by EST contigs appeared expressed compared with only $71 \%$ of those based solely on a genomic read. The size of the $P$. infestans transcriptome is not firmly established but is believed to be somewhat higher than the 15,743 and 19,027 genes predicted from the draft genomes of $P$. ramorum and $P$. sojae (Tyler et al. 2006). Therefore, the GeneChip appears to detect a majority of $P$. infestans genes.

A second assessment of the GeneChips measured the frequency of false changes between technical replicates. In all possible pair-wise comparisons between five GeneChips probed with RNA from the same hyphal sample, on average, $0.07 \%$ of genes changed by more than twofold; such genes all exhibited expression levels close to the detection threshold. It follows that approximately 11 genes may show a false change in comparisons between arrays. This rate compares favorably with data from other Affymetrix GeneChips (Zhu et al. 2001).

\section{Initial analysis of the life cycle.}

Gene expression in nonsporulating hyphae, asexual sporangia, sporangia undergoing cleavage, swimming zoospores, and germinated cysts containing appressoria were characterized using isolate 88069. Several strategies controlled for experimental error. First, replicate hybridizations were used for each tissue type, although only two replicates were employed because a limited number of the custom GeneChips were made available for this study. Second, RNAs for the two replicates were prepared by research groups in North America or Europe. Third, RNA for each hybridization was pooled from two or three independent cultures made by that group. Finally, before using an RNA sample in an experiment, the expression of selected well-characterized genes was measured to ensure that the tissue was properly prepared. The latter was necessary because transitions between most developmental stages occur rapidly in $P$. infestans. In the case of ungerminated sporangia, for example, it was prudent to ensure that sporangia had been isolated fast enough to avoid activating zoosporogenesis-associated genes because their transcripts can be induced by immersing sporangia in water for $10 \mathrm{~min}$ or less (Tani et al. 2004).

Altogether, the expression of 12,656 genes was detected in at least one of the five developmental stages based on the present calls made by the Affymetrix MAS software. Expression was detected for 8,509 genes in hyphae, 8,975 in sporangia, 8,484 in cleaving sporangia, 7,295 in zoospores, and 7,856 in the stage containing germinated cysts and appressoria. A more conservative calculation of the number of reliably expressed genes based on the coefficient of variation between replicates 
is 11,866 (Fernandes et al. 2002). The replicate RNA probes appeared to be relatively uniform because correlation coefficients were 0.96 for the two hyphal samples, 0.95 for sporangia, 0.99 for cleaving sporangia, 0.98 for zoospores, and 0.95 for germinated cysts. Median coefficients of variation were an acceptable $17.7,19.8,14.0,17.0$, and $21.3 \%$ for those samples, respectively.

As will be described in the following sections, these replicated data (after per-chip and per-gene normalization) were used to identify genes up- or downregulated at each developmental transition, and such results were validated by quantitative reverse-transcription polymerase chain reaction (qRTPCR). However, false negatives likely exist in the dataset for mRNAs showing modest changes (i.e., twofold induction) due to the limited number of replicates and biological variation between samples. That such variation exists is not surprising because subtle differences might exist in the handling of cultures by each laboratory, and media prepared from rye grain in different laboratories might vary.

\section{Overview of changes during the life cycle.}

The mRNA levels of nearly half of the genes change significantly during developmental transitions (Fig. 1). For example, when comparing sporangia to hyphae using a $P$ value threshold of $0.05,1,474$ genes are upregulated $>2$-fold and 292 by $>128$-fold; 1,492 and 97 are repressed $>2$-fold or $>128$-fold, respectively. Major changes also occur in the other developmental shifts; for example, induced by $>128$-fold are 81 genes in cleaving sporangia versus sporangia, 22 in zoospores versus cleaving sporangia, and 120 in germinated cysts versus zoospores. In a comparison of hyphae versus germinated cysts, 105 genes are upregulated $>128$-fold in the former; we did not actually examine hyphae derived from germinated cysts but this would be the natural progression of development in nature. Most genes exhibiting $>128$-fold changes are stage specific, with insignificant signals detected in some tissue types.

Although Figure 1 demonstrates that many genes change between each stage of the life cycle, the true extent of mRNA modulation is greater because numerous genes exhibit incremental changes. For example, the abundance of some mRNAs is very low in hyphae, intermediate in sporangia, and high during zoosporogenesis. When maximum fold changes over the entire life cycle are calculated, $14 \%$ of genes or 1,608 vary $>128$-fold (Fig. 2A). In all, 1,843 genes change $>100$-fold and more than half, or 7,165 genes, show $>2$-fold differences based on the $P<0.05$ criterion.

Such stepwise changes are expected due to the pattern and rapidity of development. The formation of a sporangium, for example, involves the flow of hyphal cytoplasm into an expanding terminal swelling of a sporangiophore, with a physical separation between the hypha and sporangium only established at the terminal stages of sporulation. Therefore, some transcripts synthesized exclusively within hyphae would persist within sporangia. Similarly, some modulations in mRNA populations initiated late during sporulation likely continue during cleavage, changes started during cleavage would persist through the swimming

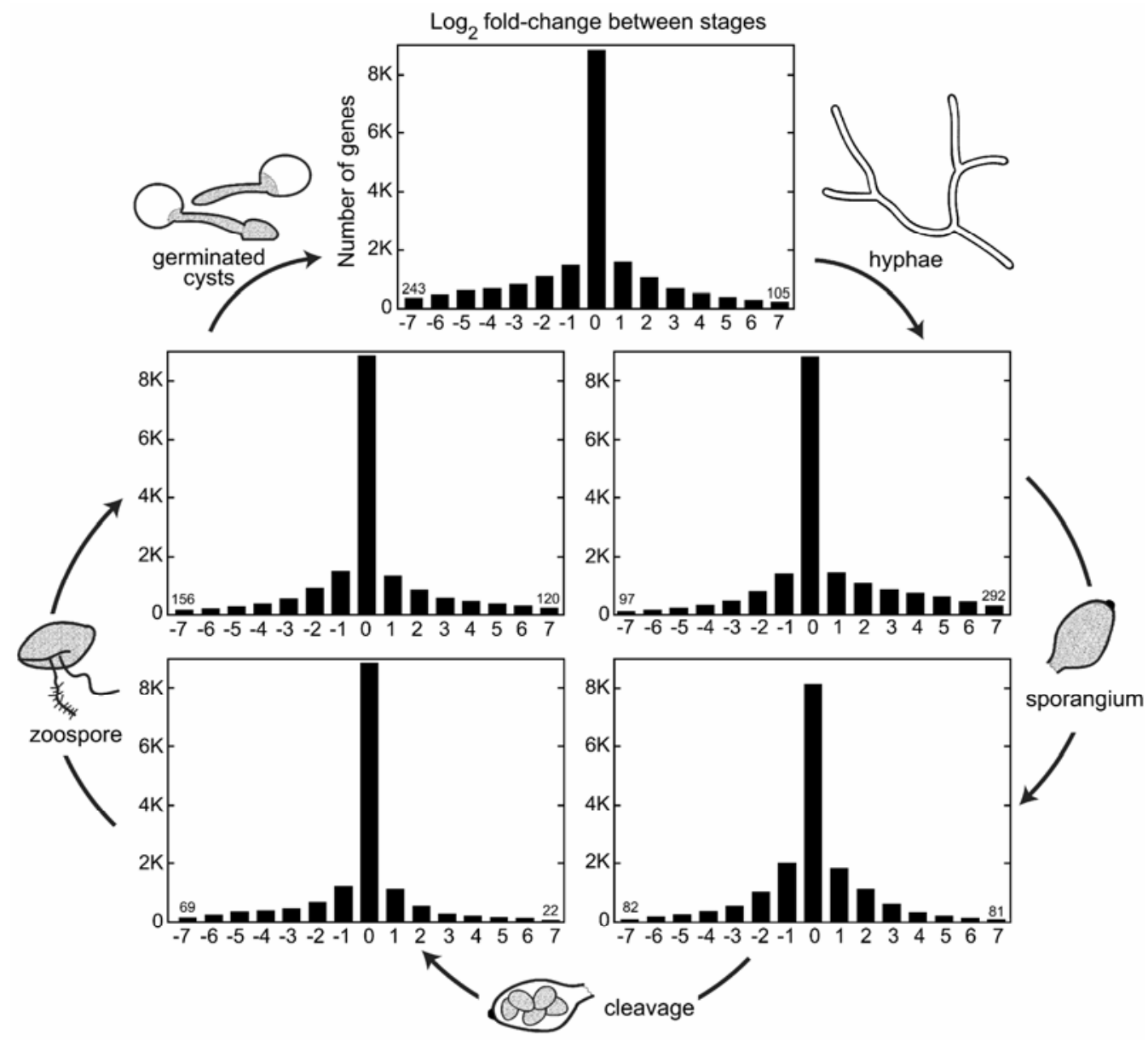

Fig. 1. Overview of expression changes during Phytophthora infestans development. Illustrated are the stages examined (hyphae, sporangia, cleavage, zoospore, and germinated cysts containing appressoria) and number of up- or downregulated genes during each transition, filtered for significance at $P<0.05$. The number of genes changing are graphed at the indicated thresholds ( 1 to 7 in $\log _{2}$ format, equivalent to 2 - to 128 -fold-change) (e.g., the bar drawn at 1 represents genes with $\geq 2$-fold or more induction). For changes of 128 -fold or more, the actual number of genes are written above the bars to aid the reader. 
zoospore stage, and germinating cysts with appressoria are likely to produce transcripts required for both successful penetration and later stages of host colonization.

Some of these relationships are reflected in the condition tree in Figure 2B. This shows that the mRNA profile of hyphae is closest to that of sporangia, and zoospores are nearest to cleaving sporangia. The stages containing germinating cysts and appressoria cluster with hyphae and sporangia, which may reflect their shared patterns of polarized growth (sporangia also can extend hyphae through direct germination) and the preparation of germinated cysts to assimilate external nutrients, as do hyphae. The fact that cleaving sporangia are not closest to sporangia, even though separated by only $1 \mathrm{~h}$ of cold treatment, probably reflects two issues. The first is a need to synthesize factors for post-zoospore stages such as cyst germination. The second is a commitment of the cleaving sporangium away from hyphal growth; prior to cleavage, sporangia retain

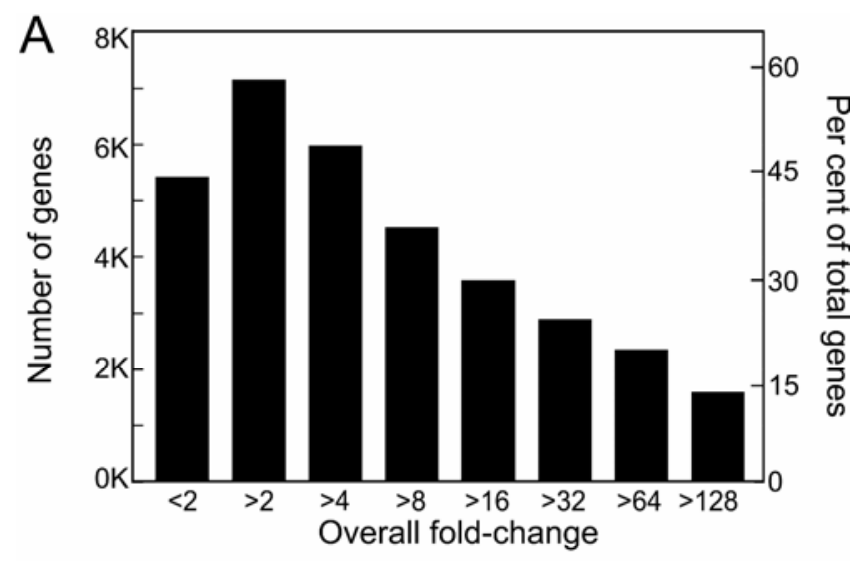

B

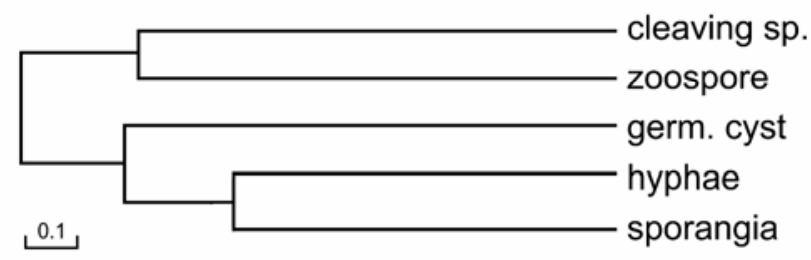

Fig. 2. Changes and similarities between developmental stages. A, Number of genes showing changes in mRNA levels at the indicated thresholds. Foldchange values represent the maximum difference between developmental stages, after filtering for differences significant at $P<0.05$. B, Condition tree determined using the Pearson correlation method with average linkage. an ability to extend hyphal-like germ tubes (e.g., direct germination). The ability to germinate directly is inhibited by cold treatment, which may trigger the elimination of mRNAs associated with hyphal growth (Tani and Judelson 2006). In the future, it will be important to assess the relative contributions of changes in de novo transcription versus mRNA stability in this and other developmental transitions.

\section{Validation of GeneChip data by qRT-PCR.}

qRT-PCR assays demonstrated the robustness of the GeneChip data. This involved testing 74 genes changing between developmental stages, using RNA preparations independent from those used with the GeneChips. The genes examined, which are listed in Supplementary Table 1, were selected to represent a range of induction ratios at each life-stage transition (usually from approximately 3 - to $>100$-fold).

General agreement was observed between the GeneChip and qRT-PCR results, with overall calculated fold-change values well correlated (mean correlation coefficient $R$ for the five developmental transitions was 0.78) (Fig. 3). The extent of induction of individual genes often varied, however. For example, gene Pi001985 showed only $5.2 \pm 0.5$-fold induction during cleavage based on GeneChip data while $23 \pm 2$-fold induction was calculated from qRT-PCR. Also, whereas $98 \%$ of tested genes were confirmed to be induced by qRT-PCR, an exception was Pi003939, for which GeneChip and RT-PCR data indicated $3.8 \pm 0.2$ - and $1.7 \pm 1.0$-fold ratios in sporangia versus hyphae, respectively; the latter does not represent statistically significant induction. Technical issues responsible for differences between array and qRT-PCR data are well described (Etienne et al. 2004) although, in this case, biological variation between samples used for GeneChip and qRT-PCR studies is likely the most significant factor because induction ratios are highly dependent on the precise time of sampling, age of spores, and so on.

\section{Clusters of transcript profiles during development.}

Expression patterns of genes ordered on the basis of hierarchical clustering are portrayed in the left side of Figure 4, where red and green coloration symbolize up- and downregulation, respectively, compared with the mean of the five stages. The right side of the figure illustrates average expression patterns within 11 selected developmentally regulated clusters (A to K). Twenty genes from each group are described in Table 2. These were selected by first ranking the genes within each of the 11 clusters in decreasing order of fold change during development (peak to valley), and then selecting the top 20 with either similarity to non-oomycete proteins (using
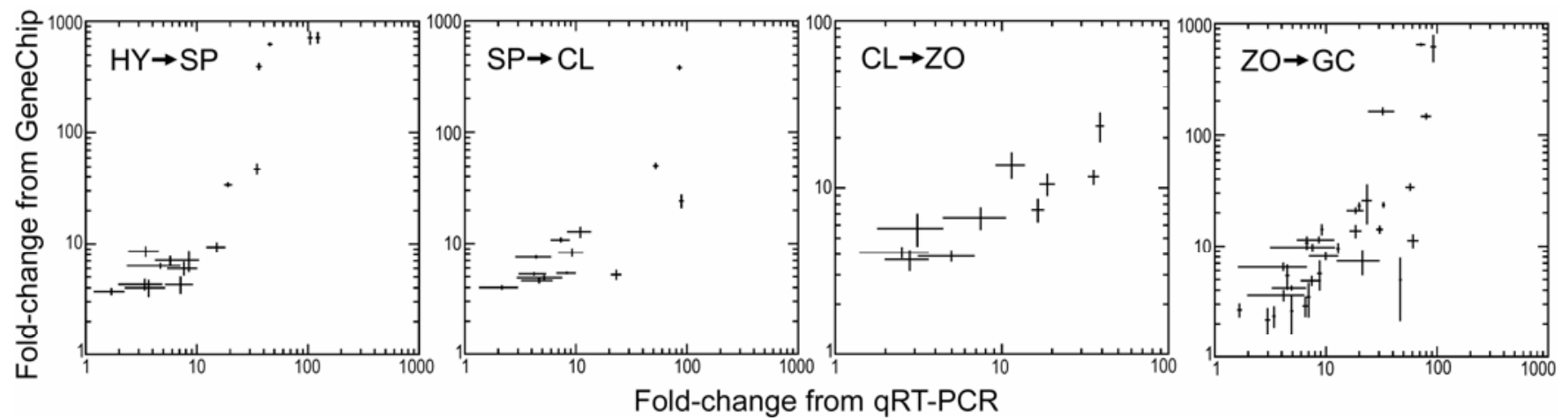

Fig. 3. Validation of microarray data by quantitative reverse-transcription polymerase chain reaction (qRT-PCR). Graphed are fold-change values for 74 genes in comparisons between hyphae (HY), sporangia (SP), cleaving sporangia (CL), swimming zoospores (ZO), and the stage with germinated cysts and appressoria (GC). For example, the HY $\rightarrow$ SP panel shows the SP/HY ratio. qPCR was performed based on the $\Delta \Delta \mathrm{C}_{\mathrm{T}}$ method. Error bars are calculated from the two replicate GeneChip studies and three replicate qRT-PCR assays. 
BLASTX with an $E$ value cutoff of $10^{-5}$ ) or previously characterized oomycete-specific proteins.

The fraction of genes within each group resembling conserved non-oomycete-specific proteins ranged from 14 to $66 \%$. This was determined from analyses of 100 genes within each of groups A to $\mathrm{K}$ using full-length open reading frames derived from whole-genome data, which became available toward the end of this study from the Broad Institute of MIT and Harvard. By comparison, $69 \%$ of genes not changing between stages encode conserved proteins. Of genes expressed mostly in hyphae but not spores (groups C, D, E, and F), 48\% match conserved proteins compared with $25 \%$ in spore-induced groups (A, B, $\mathrm{G}, \mathrm{H}, \mathrm{I}, \mathrm{J}$, and $\mathrm{K}$ ). Therefore, developmentally regulated genes are more likely to be oomycete specific, particularly those specific to spore stages. Many genes lacking hits in BLASTX are predicted to encode structural proteins because they have extensive low-complexity regions or abundant glycosylation sites (Lagow et al. 1999; Sachetto-Martins et al. 2000). Other developmentally regulated genes encode cellular regulators, metabolic enzymes, transporters, or participants in plant pathogenesis. Examples from the 11 groups will be described in the following sections; the complete dataset can be extracted from the NCBI GEO archive.

\section{Groups expressed predominantly in sporangia.}

Two groups ( $\mathrm{G}$ and $\mathrm{H}$, with 324 and 629 members, respectively) represent mRNAs that are very low in abundance in hyphae, high in sporangia, and fall in concentration through the germinated cyst stage. These are distinguished by whether their mRNAs are mostly exclusive to sporangia $(G)$ or also persist throughout cleavage $(\mathrm{H})$. The difference in maintenance of the mRNAs during cleavage, which may result from variation in de novo transcription or mRNA stability, may be a clue to their function. For example, group $\mathrm{G}$ might participate in sporangia formation and group $\mathrm{H}$ in zoospores. Consistent with this premise is the observation that 9 of the top 20 genes in group $\mathrm{H}$ encode flagellar components, such as various dyneins and flagellar root or basal body proteins (Pazour et al. 2005). Also in group $\mathrm{H}$ are other possible participants in zoospore biology such as an adaptin; such proteins regulate the dynamin-dependent release of coated vesicles from membrane surfaces and might control the exocytosis of cell wall material during encystment or membrane reorganization during zoospore formation.

The detection of zoospore-related proteins in group $\mathrm{H}$ might appear to contradict the hypothesis that zoosporogenesis and encystment occur solely using RNA and protein stored in sporangia, which was inferred from inhibitor studies (Clark et al. 1978; Penington et al. 1989). Those studies showed, for example, that adding actinomycin D to sporangia strongly reduced RNA synthesis but not zoospore release. However, the question of whether such inhibitors enter sporangia rapidly enough to block mRNA synthesis before zoosporogenesis starts was raised by a study that found that transcription of the zoosporogenesis-specific PiPkzl gene was not totally arrested by high concentrations $(100 \mu \mathrm{g} / \mathrm{ml})$ of actinomycin D (Judelson and Roberts 2002). Nevertheless, it seems reasonable to conclude that a substantial reduction in transcription is insufficient to block zoospore formation. Although it can not be excluded that some de novo transcription is required for zoosporogenesis, a plausible explanation for the continued synthesis of mRNAs for zoospore components late during cleavage (and in zoospores) is that this enhances the fitness of zoospores. In species such as Chlamydomonas, active flagella were shown to be dynamic structures that incorporate new protein from cytoplasm (Song and Dentler 2001). Assuming that the same is true in Phytophthora spp., continued synthesis of mRNA and protein for flagellar com-
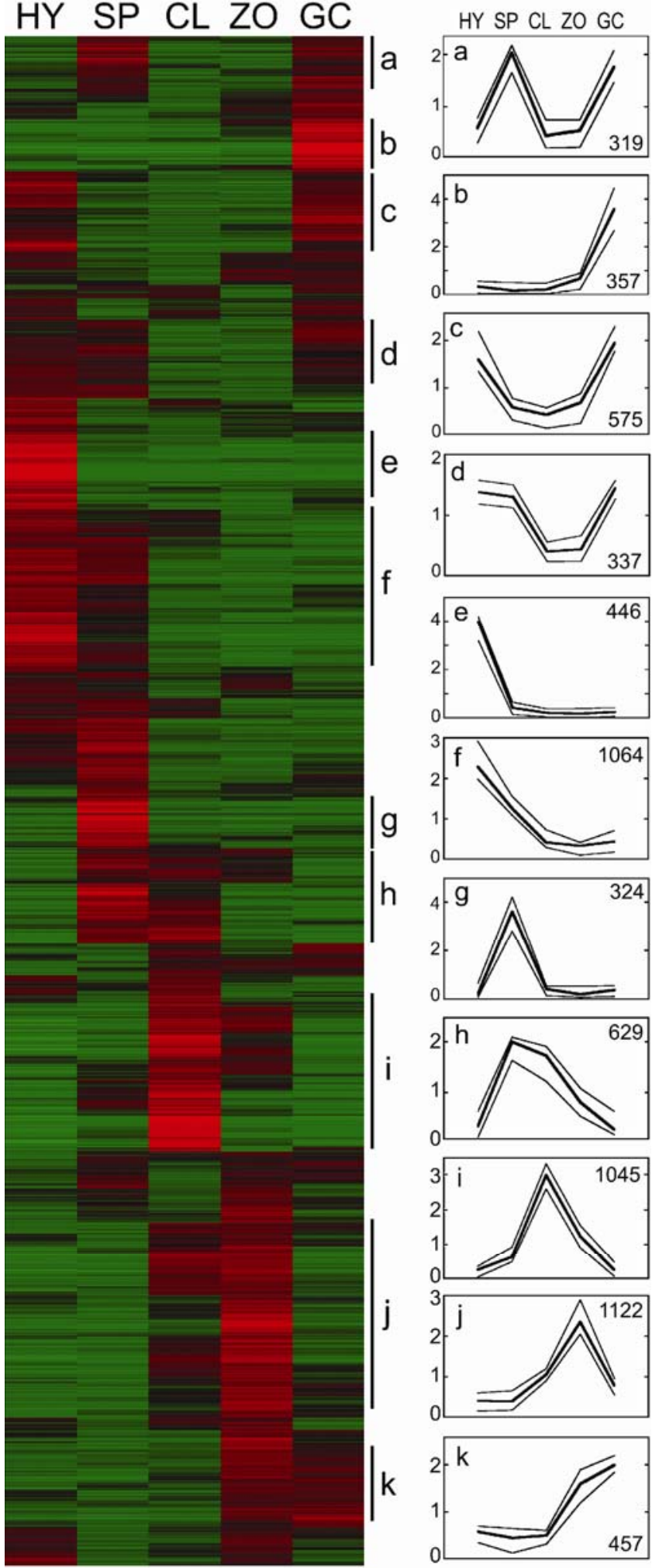

e
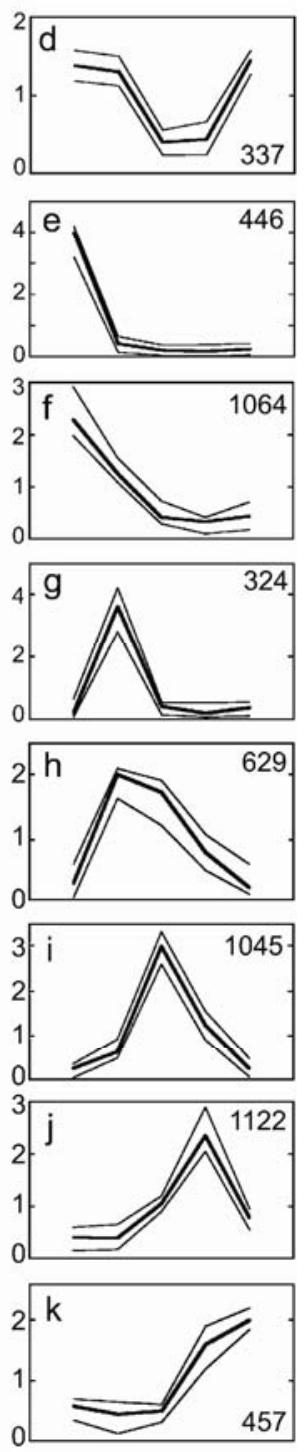

Fig. 4. Hierarchical clustering of microarray data. The right panel indicates expression levels of genes in hyphae (HY), sporangia (SP), cleaving sporangia (CL), swimming zoospores (ZO), and germinating zoospore cysts plus appressoria (GC). Data are clustered based on Pearson correlations with average linkage, using data subjected to per-chip and then per-gene normalization. Red represents upregulated compared with the mean of all five stages, and green represents downregulation. Lines mark notable major groups (a to k). Shown in the right image are expression profiles of genes within those groups. The center line represents average expression, with upper and lower lines representing upper and lower quartiles. The numbers of genes within each cluster are indicated in right of each panel. 
ponents would be expected to increase the longevity of the motile stage.

Groups $\mathrm{G}$ and $\mathrm{H}$ also encode many putative regulators, with a GTP-binding protein, a pumilio family RNA-binding protein, a protein kinase, two DNA-binding proteins, and Cdc14 protein phosphatase (Table 2). The presence of the latter in group $\mathrm{H}$ illustrates that divining function from expression kinetics is not straightforward: the Cdc14 mRNA profile is similar to that of most flagellar components, yet gene silencing showed that Cdc14 is needed very early during sporulation (Ah Fong and Judelson 2003). However, Cdc14 may continue to be needed in the zoospore or cyst stages, perhaps to maintain mitotic dormancy (Krasinska et al. 2007). A more trivial explanation is that Cdc14 mRNA is very long lived and persists long after the cognate protein played its role.

mRNA levels of group A (319 members) are also low in hyphae and induced at sporulation, but drop quickly during zoosporogenesis and rise in the stage containing germinated cysts with appressoria. Predicting the function of these genes is difficult without excessive speculation about physiological states shared by sporangia and germinating cysts. However, it is interesting that 3 of the 20 matches shown in Table 2 are to retroelements. The retroelement-like sequences appear to be nonfunctional due to frame shifts or truncations, but still could contain a functional promoter. Stress experienced during development might be inducing such elements, as in other sys-

Table 2. Top 20 genes in expression groups having informative BLAST matches

\begin{tabular}{|c|c|c|c|c|c|c|c|c|}
\hline \multirow[b]{2}{*}{ Rank $^{\mathbf{b}}$} & \multirow[b]{2}{*}{ Gene } & \multicolumn{5}{|c|}{ Normalized expression $^{a}$} & \multirow[b]{2}{*}{ Best BLASTX match } & \multirow[b]{2}{*}{$E$ value $^{\mathrm{d}}$} \\
\hline & & HY & SP & $\mathbf{C L}$ & $\mathbf{Z O}$ & GC & & \\
\hline \multicolumn{9}{|c|}{$\overline{\text { Group A }(20 \%)^{\mathrm{e}}}$} \\
\hline 1 & Pi008046 & 0.0 & 21.6 & 0.0 & 0.0 & 23.3 & Retroelement polyprotein, Oryza japonica CAE02978 & $7 e-60$ \\
\hline 2 & Pi014042 & 0.0 & 3.0 & 0.0 & 0.0 & 3.9 & Retroelement polyprotein, Phytophthora infestans AAV92918 & $2 \mathrm{e}-57$ \\
\hline 6 & Pi007454 & 0.0 & 1.6 & 0.7 & 0.3 & 1.2 & Ankyrin-like, Strongylocentrotus purpuratus XP_798904 & $3 e-12$ \\
\hline 7 & $\mathrm{Pi} 003902$ & 0.0 & 1.4 & 0.1 & 0.1 & 0.8 & hsp70, Nicotiana tabacum AAR17080 & 0.0 \\
\hline 19 & Pi006746 & 0.0 & 0.5 & 0.0 & 0.0 & 0.3 & Retroelement polyprotein, $P$. infestans CAI72306 & $1 \mathrm{e}-14$ \\
\hline 23 & Pi013112 & 0.0 & 0.6 & 0.3 & 0.2 & 0.5 & Microtubule binding protein, Aedes aegypti EAT34524 & $7 e-09$ \\
\hline 34 & Pi002505 & 0.0 & 0.8 & 0.3 & 0.4 & 0.9 & Nuclear pore complex protein, Leishmania major CAJ05760 & $3 e-16$ \\
\hline 35 & Pi008909 & 0.0 & 0.5 & 0.0 & 0.0 & 0.4 & Aquaporin, Anguilla anguilla CAH04573 & $5 e-48$ \\
\hline 41 & Pi002329 & 0.0 & 0.8 & 0.0 & 0.0 & 0.3 & Plectin, Homo sapiens AAB05427 & $2 \mathrm{e}-10$ \\
\hline 48 & Pi013713 & 0.3 & 5.2 & 1.1 & 1.2 & 9.8 & Carnitine palmitoyltransferase, Rattus norvegicus AAH72522 & 0.0 \\
\hline 51 & Pi011665 & 0.2 & 3.1 & 0.8 & 0.0 & 2.0 & cAMP-dependent protein kinase, Gonyaulax polyedra AAA64341 & $3 e-91$ \\
\hline 54 & Pi013572 & 0.6 & 8.9 & 2.2 & 1.1 & 6.7 & Tyrosine aminotransferase, Danio rerio XP_689908 & $4 \mathrm{e}-63$ \\
\hline 56 & Pi014369 & 0.1 & 0.1 & 0.4 & 0.2 & 2.0 & Chromosome segregation ATPase, H. sapiens AAL11632 & $8 \mathrm{e}-11$ \\
\hline 59 & Pi012581 & 0.1 & 0.7 & 0.1 & 0.3 & 1.1 & RNA (guanine-9-)methylttansferase, Trypanosoma cruzi EAN94865 & $2 \mathrm{e}-18$ \\
\hline 61 & Pi002592 & 0.4 & 5.0 & 1.0 & 1.0 & 7.1 & Protein phosphatase, Tribolium castaneum XP_975521 & $3 e-28$ \\
\hline 66 & Pi011712 & 0.0 & 0.5 & 0.0 & 0.0 & 0.4 & Amino acid transporter, Dictyostelium discoideum EAL61840 & $2 \mathrm{e}-58$ \\
\hline 68 & Pi002586 & 10.5 & 98.9 & 3.4 & 1.0 & 73.5 & hsp101 chaperone, D. discoideum XP_635137 & 0.0 \\
\hline 79 & $\mathrm{Pi} 013477$ & 0.3 & 2.4 & 0.7 & 0.5 & 1.4 & Adenylate cyclase, Myxococcus xanthus ABF90960 & $1 \mathrm{e}-24$ \\
\hline 85 & Pi005747 & 0.6 & 4.0 & 0.5 & 0.6 & 2.9 & Dehydrorhamnose reductase, $O$. sativa AAS01025 & $4 \mathrm{e}-19$ \\
\hline 101 & Pi003256 & 0.8 & 4.0 & 0.6 & 0.1 & 2.3 & Leucine-rich repeat protein, S. purpuratus XP_793918 & 1e-17 \\
\hline \multicolumn{9}{|c|}{ Group B $(44 \%)$} \\
\hline 1 & Pi005201 & 0.0 & 0.5 & 1.8 & 3.5 & 77.5 & RXLR protein & na \\
\hline 3 & Pi006108 & 0.0 & 0.0 & 0.0 & 3.8 & 43.5 & avr1B-like RXLR protein, $P$. sojae AAM20935 & $1 \mathrm{e}-135$ \\
\hline 4 & $\mathrm{Pi} 000144$ & 0.0 & 0.0 & 0.1 & 0.8 & 37.6 & ipiB-like protein, $P$. infestans AAY 43375 & $2 e-31$ \\
\hline 5 & Pi009097 & 0.0 & 0.0 & 0.0 & 1.1 & 22.2 & RXLR protein & na \\
\hline 7 & Pi000059 & 0.1 & 0.4 & 4.8 & 38.3 & 114.2 & Cutinase, Kineococcus radiotolerans EAM73881 & $4 \mathrm{e}-38$ \\
\hline 12 & Pi002924 & 0.0 & 0.0 & 0.2 & 2.4 & 14.2 & Hydroxyproline-rich glycoprotein, $O$. sativa CAA 43583 & $1 e-9$ \\
\hline 13 & Pi007849 & 0.0 & 0.0 & 0.0 & 0.2 & 13.0 & RXLR protein & na \\
\hline 17 & Pi001501 & 0.0 & 0.6 & 1.3 & 4.6 & 11.9 & Glutathione S-transferase, Tetrahymena thermophila XP 001009875 & $2 \mathrm{e}-26$ \\
\hline 23 & Pi010090 & 0.0 & 0.3 & 0.1 & 0.5 & 8.0 & Retroelement polyprotein, $O$. sativa AAP53043 & 0.0 \\
\hline 27 & $\mathrm{Pi} 003485$ & 0.0 & 0.1 & 0.7 & 0.0 & 6.8 & $\beta-1,4-X y l a n a s e$, Aspergillus fumigatus EAL89199 & $4 \mathrm{e}-52$ \\
\hline 37 & Pi005101 & 0.0 & 0.1 & 0.1 & 0.1 & 5.1 & ser/thr-rich wall adhesion protein, Candida albicans XP_714666 & $7 e-16$ \\
\hline 38 & Pi003811 & 0.0 & 0.6 & 0.0 & 0.3 & 5.0 & Calmodulin-like protein kinase, Toxoplasma gondii ABA 60892 & $1 \mathrm{e}-23$ \\
\hline 43 & Pi005244 & 0.0 & 0.0 & 0.0 & 0.0 & 4.3 & Serine/thronine protein kinase, $O$. sativa AAO72550 & $7 e-28$ \\
\hline 48 & $\mathrm{Pi} 006384$ & 0.0 & 0.0 & 0.0 & 0.0 & 3.4 & bZIP transcription factor, $P$. infestans AAX19188 & $4 \mathrm{e}-28$ \\
\hline 53 & $\mathrm{Pi} 007050$ & 0.2 & 0.2 & 0.5 & 21.2 & 70.8 & Protease, Oceanicaulis alexandrii EAP91021 & $1 \mathrm{e}-18$ \\
\hline 57 & Pi001883 & 0.1 & 0.7 & 0.7 & 1.8 & 13.7 & Bifunctional catalase-peroxidase, Escherichia coli AAC70085 & $2 \mathrm{e}-113$ \\
\hline 61 & $\mathrm{Pi} 015336$ & 0.0 & 0.0 & 0.0 & 0.0 & 2.1 & Protein kinase, Paramecium tetraurelia CAK64916 & $1 \mathrm{e}-27$ \\
\hline 62 & Pi010868 & 0.1 & 0.4 & 3.9 & 13.7 & 30.7 & Zinc ion transporter, A. fumigatus EAL 87480 & $2 e-21$ \\
\hline 71 & $\mathrm{Pi} 002242$ & 0.2 & 0.1 & 0.0 & 0.6 & 29.2 & Aquaporin $10, H$. sapiens AAH69607 & $3 e-43$ \\
\hline 76 & Pi006838 & 0.1 & 0.0 & 0.7 & 2.8 & 12.3 & Calcium-chelating protein, Moorella thermoacetica YP_430550 & $5 e-11$ \\
\hline
\end{tabular}

\footnotetext{
a Based on per-chip normalization in hyphae (HY), sporangia (SP), cleaving sporangia (CL), zoospore (ZO), and germinated cysts and appressoria (GC), with the average $P$. infestans gene set to 1.0.

${ }^{\mathrm{b}}$ Genes were sorted based on decreasing overall fold change within each cluster. The numbers shown match the position of each gene on that list, with ranks not shown corresponding to genes lacking significant BLASTX hits.

${ }^{\mathrm{c}}$ Matches are shown for the 20 sequences within each group having the greatest fold changes during development and an informative BLASTX hit. These were determined using an $E$ value threshold of $10^{-5}$, and excludes hits against conserved proteins with no apparent function. Except for oomycete-specific genes and Phytophthora genes previously studied in detail, the best non-oomycete match is listed. Genes annotated as "RXLR protein" contain the RXLR/dEER motif which is associated with effectors believed to be transported from Phytophthora into plant cells (Birch et al. 2006). Results are based on predicted full-length sequences, and unigenes having similar annotations were cross-checked using the full-length sequences to exclude redundancy.

${ }^{\mathrm{d}}$ na $=$ Not applicable.

${ }^{\mathrm{e}}$ The percentage indicated in parentheses for each group is the percentage of sequences having matches in GenBank, based on analyses of the 100 genes having the greatest overall fold changes.
} 
tems (Todeschini et al. 2005). The induction of two heat-shock proteins, a protein that mobilizes lipids to $\beta$-oxidation (carnitine acyltransferase), and a methionine salvage enzyme (tyrosine aminotransferase) may be additional signals that stress is perceived at these stages.
An alternative explanation for the induction of the retroelement-like sequences may be the distribution of cryptic sites for a developmentally regulated transcription factor throughout the genome or the insertion of retroelements near inducible promoters. In any case, it follows that some stage-specific

Table 2. (continued from preceding page)

\begin{tabular}{|c|c|c|c|c|c|c|c|c|}
\hline \multirow[b]{2}{*}{ Group, rank $^{\mathbf{b}}$} & \multirow[b]{2}{*}{ Gene } & \multicolumn{5}{|c|}{ Normalized expression ${ }^{a}$} & \multirow[b]{2}{*}{ Best BLASTX match ${ }^{c}$} & \multirow[b]{2}{*}{$E$ value $^{d}$} \\
\hline & & HY & SP & $\mathbf{C L}$ & $\mathbf{Z O}$ & GC & & \\
\hline \multicolumn{9}{|l|}{ Group C $(46 \%)$} \\
\hline 2 & Pi014776 & 15.9 & 0.0 & 0.0 & 1.9 & 43.0 & Glucose transporter, A. aegypti EAT44948 & $3 e-47$ \\
\hline 3 & Pi002520 & 32.6 & 0.3 & 0.0 & 0.8 & 21.5 & Acid phosphatase, Neurospora crassa XP_958715 & $6 e-57$ \\
\hline 4 & Pi011385 & 23.5 & 0.0 & 0.0 & 0.1 & 20.3 & myb transcription factor, Hyaloperonospora parasitica AAY58905 & $6 e-11$ \\
\hline 5 & Pi004777 & 17.1 & 0.0 & 0.0 & 0.0 & 13.5 & Amino acid transporter, Ustilago maydis XP_758306 & $8 \mathrm{e}-11$ \\
\hline 8 & Pi004718 & 24.0 & 4.3 & 0.0 & 0.8 & 25.7 & RXLR protein & na \\
\hline 13 & Pi003621 & 5.1 & 0.1 & 0.0 & 0.0 & 4.5 & Drug/ion antiporter, Mus musculus NP_001028 & $2 e-29$ \\
\hline 22 & $\mathrm{Pi000048}$ & 1.2 & 0.0 & 0.0 & 0.0 & 2.4 & Kazal serine protease inhibitor, $P$. infestans AAT00501 & $3 e-84$ \\
\hline 23 & $\mathrm{Pi006028}$ & 1.5 & 0.0 & 0.0 & 0.0 & 2.4 & myb transcription factor, $H$. parasitica AAY58905 & $6 e-11$ \\
\hline 27 & Pi006477 & 1.5 & 0.1 & 0.0 & 0.0 & 2.1 & $\beta$-1,3-endoglucanase, A. fumigatus AF121133 & $4 e-79$ \\
\hline 32 & Pi013422 & 11.8 & 0.6 & 0.1 & 0.0 & 11.2 & Amino acid transporter, O. sativa BAB62326 & $2 \mathrm{e}-52$ \\
\hline 42 & Pi006139 & 1.5 & 0.0 & 0.0 & 0.0 & 0.9 & MAP protein kinase, A. thaliana AAM20478 & $2 \mathrm{e}-38$ \\
\hline 48 & Pi005406 & 71.4 & 0.7 & 0.7 & 1.5 & 52.3 & Calcium chelating serine protease, Nitrosococcus oceani ABA57867 & $7 e-17$ \\
\hline 50 & Pi004105 & 1.1 & 0.1 & 0.0 & 0.4 & 0.7 & Protein lysine methyltransferase, Pisum sativum Q43088 & $3 e-20$ \\
\hline 55 & Pi004009 & 15.3 & 0.1 & 0.5 & 3.4 & 26.9 & $\beta$-1,3-Exoglucanase, S. cerevisiae AAS56427 & $9 e-23$ \\
\hline 56 & Pi006700 & 6.1 & 0.0 & 0.1 & 0.1 & 5.1 & RNA polymerase CTD interacting factor, Bos taurus XP_615579 & $4 e-22$ \\
\hline 62 & Pi004408 & 0.6 & 0.5 & 0.1 & 0.0 & 2.3 & Calcium-dependent protein kinase, Plasmodium falciparum Q8ICR0 & $2 e-51$ \\
\hline 63 & Pi003901 & 1.4 & 0.1 & 0.1 & 0.0 & 5.4 & Phosphoglycerate kinase, Volvox carteri AF110782 & 0.0 \\
\hline 66 & Pi005235 & 3.8 & 0.9 & 0.3 & 0.0 & 12.1 & Sugar symporter, $S$. purpuratus XP_787113 & $3 e-23$ \\
\hline 71 & $\mathrm{Pi002936}$ & 35.3 & 0.9 & 1.0 & 3.6 & 33.7 & ABC transporter, A. thaliana AAC31858 & $2 \mathrm{e}-27$ \\
\hline 73 & $\mathrm{PiO02234}$ & 5.3 & 0.9 & 0.1 & 1.0 & 3.8 & Tryptophanyl-tRNA synthetase, D. discoideum XP_645983 & $6 \mathrm{e}-138$ \\
\hline \multicolumn{9}{|l|}{$\mathrm{D}(40 \%)$} \\
\hline 2 & Pi000908 & 6.4 & 4.9 & 0.0 & 0.3 & 2.9 & COP9 signalosome subunit, X. laevis AAH81245 & $2 e-21$ \\
\hline 4 & Pi007605 & 0.6 & 1.5 & 0.0 & 0.1 & 1.4 & Retroelement polyprotein, Zea mays AAN40030 & $3 e-16$ \\
\hline 8 & Pi001785 & 1.2 & 1.2 & 0.0 & 0.0 & 0.8 & Zinc finger DNA-binding protein, Xenopus laevis AAH77329 & $2 e-65$ \\
\hline 11 & $\mathrm{Pi001022}$ & 8.2 & 6.2 & 0.1 & 1.3 & 4.9 & DNA damage-binding protein, Lycopersicon cheesmanii Q6E7D1 & 0.0 \\
\hline 13 & $\mathrm{Pi012482}$ & 1.2 & 1.2 & 0.0 & 0.0 & 1.7 & Glutamine amidotransferase, D. discoideum AAO51647 & 0.0 \\
\hline 15 & $\mathrm{PiO04280}$ & 5.3 & 5.9 & 0.1 & 1.4 & 5.6 & Serine-type peptidase, $A$. thaliana NP_568490 & 0.0 \\
\hline 16 & Pi001503 & 1.6 & 1.4 & 0.0 & 0.1 & 1.0 & GTPase regulator/RAB, M. musculus NP_061288 & $2 \mathrm{e}-37$ \\
\hline 17 & Pi009197 & 0.2 & 0.3 & 0.0 & 0.0 & 0.6 & Ankyrin domain protein, Wolbachia pipientis AAW23170 & $2 e-22$ \\
\hline 20 & Pi011120 & 2.5 & 1.6 & 0.1 & 0.2 & 3.7 & G protein-coupled receptor, S. purpuratus XP_796821 & $6 e-43$ \\
\hline 23 & Pi013188 & 0.6 & 0.5 & 0.0 & 0.1 & 0.5 & DNA binding protein, Medicago truncatula ABD33215 & $4 e-26$ \\
\hline 25 & Pi001843 & 1.1 & 1.4 & 0.1 & 0.4 & 2.2 & Membrane anchored protease, Solibacter usitatus ZP_0052446 & $7 e-95$ \\
\hline 26 & Pi003596 & 0.7 & 0.5 & 0.0 & 0.0 & 0.4 & Nuclear growth factor binding-protein, D. rerio AAH54612 & $1 e-35$ \\
\hline 39 & $\mathrm{PiO02174}$ & 1.5 & 1.9 & 0.1 & 0.3 & 2.1 & tRNA-ribosyltransferase, $O$. sativa $\mathrm{BAF} 25348$ & $2 \mathrm{e}-127$ \\
\hline 42 & Pi002320 & 36.4 & 47.7 & 4.1 & 10.5 & 58.9 & Putative sugar transporter, A. thaliana $\mathrm{NP}_{-} 188628$ & $1 e-51$ \\
\hline 43 & $\mathrm{Pi005496}$ & 7.9 & 11.4 & 0.8 & 0.2 & 12.1 & Acyl-CoA synthetase, Nostoc punctiforme ZP_001098 & $6 e-104$ \\
\hline 47 & $\mathrm{Pi000958}$ & 2.5 & 3.4 & 0.4 & 0.8 & 5.3 & Arginyl-tRNA synthetase, Synechococcus elongatus ABB57607 & $3 e-89$ \\
\hline 48 & $\mathrm{Pi0} 15475$ & 1.9 & 1.7 & 0.2 & 0.9 & 2.7 & G1 cyclin, A. gossypii AAS52514 & $4 e-34$ \\
\hline 61 & $\mathrm{Pi002692}$ & 1.3 & 1.5 & 0.3 & 0.0 & 2.4 & Cysteine synthase, S. pombe CAB 08745 & $6 e-61$ \\
\hline 62 & Pi002391 & 14.0 & 18.2 & 2.0 & 5.5 & 18.4 & ATP-dependent protease, $A$. thaliana NP_568490 & 0.0 \\
\hline 63 & Pi001470 & 2.8 & 2.5 & 0.3 & 0.1 & 2.6 & cdc2-like protein kinase, D. discoideum XP_636601 & $1 e-48$ \\
\hline \multicolumn{9}{|l|}{ Group E $(38 \%)$} \\
\hline 1 & $\mathrm{Pi004565}$ & 33.2 & 0.0 & 0.1 & 0.6 & 0.1 & Sugar transporter, A. fumigatus XP_785115 & 0.0 \\
\hline 2 & $\mathrm{PiO04146}$ & 23.3 & 0.0 & 0.0 & 0.0 & 0.0 & $\beta$-Glucosidase, $A$. thaliana $\mathrm{NP}_{-} 191573$ & $1 \mathrm{e}-72$ \\
\hline 3 & $\mathrm{Pi004295}$ & 13.4 & 0.0 & 0.0 & 0.9 & 0.0 & Mucin-like protein, Drosophila melanogaster AAF50957 & $1 e-09$ \\
\hline 4 & Pi000667 & 35.3 & 0.0 & 0.0 & 0.0 & 0.0 & Flavin oxidoreductase, Anabaena variabilis YP_325252 & $6 e-73$ \\
\hline 6 & Pi000001 & 12.2 & 0.0 & 0.0 & 0.0 & 0.0 & Necrosis-inducing protein NPP1, P. infestans AAY43363 & $2 \mathrm{e}-115$ \\
\hline 8 & Pi015868 & 11.3 & 0.0 & 0.0 & 0.0 & 0.1 & Elicitin INF1, P. infestans AAV92919 & $1 e-48$ \\
\hline 10 & Pi003715 & 29.1 & 0.0 & 0.8 & 0.6 & 1.1 & Fatty acid desaturase, Mortierella alpina Q9Y8H5 & $1 e-98$ \\
\hline 12 & Pi000585 & 6.9 & 0.0 & 0.0 & 0.0 & 0.1 & Pyruvate phosphate dikinase, A. thaliana NP_001078396 & 0.0 \\
\hline 15 & $\mathrm{Pi003814}$ & 6.1 & 0.0 & 0.6 & 0.0 & 0.0 & Putative hydrolase, $R$. norvegicus XP_220745 & $4 e-38$ \\
\hline 17 & Pi003943 & 5.6 & 0.0 & 0.0 & 0.0 & 0.0 & Cytochrome P450 monooxygenase, Glycine max ABC68403 & $5 e-59$ \\
\hline 28 & Pi004421 & 3.6 & 0.0 & 0.0 & 0.0 & 0.0 & Alkaline phosphatase, Gigaspora margarita BAD07013 & $2 \mathrm{e}-58$ \\
\hline 31 & Pi001128 & 6.8 & 0.0 & 0.0 & 0.0 & 0.0 & NAD oxidoreductase, Azotobacter vinelandii ZP_0120756 & $6 e-49$ \\
\hline 35 & Pi000086 & 2.5 & 0.0 & 0.0 & 0.0 & 0.0 & Divalent heavy-metal transporter, Candidatus kuenenia CAJ71209 & $2 e-26$ \\
\hline 37 & $\mathrm{Pi001552}$ & 2.1 & 0.0 & 0.4 & 0.00 & 0.1 & NADH:flavin oxidoreductase, A. thaliana C86227 & $2 e-68$ \\
\hline 38 & Pi002993 & 2.1 & 0.0 & 0.0 & 0.1 & 0.0 & ATP sulfurylase, Ciona intestinalis BAB00629 & 0.0 \\
\hline 42 & $\mathrm{PiO04478}$ & 6.2 & 0.0 & 0.0 & 1.7 & 0.6 & Cytochrome P450 enzyme, A. thaliana AF013293 & $1 e-65$ \\
\hline 47 & $\mathrm{Pi000045}$ & 63.0 & 0.4 & 0.0 & 1.9 & 6.8 & Peroxidase-like protein, Magnaporthe grisea XP_367967 & $8 e-19$ \\
\hline 51 & Pi000587 & 73.1 & 0.5 & 2.0 & 1.2 & 6.7 & Fatty acid elongase, Thalassiosira pseudonana AAV67799 & $2 \mathrm{e}-48$ \\
\hline 52 & Pi000913 & 28.6 & 0.2 & 0.1 & 0.0 & 0.0 & Cytochrome P450 monooxygenase, G. $\max$ CYP86A24 & $5 e-59$ \\
\hline \multirow[t]{2}{*}{54} & Pi015263 & 1.1 & 0.0 & 0.0 & 0.0 & 0.0 & Serine carboxypeptidase, A. thaliania NP_197689 & $3 e-69$ \\
\hline & & & & & & & & (continued on next page) \\
\hline
\end{tabular}


transcripts may not actually play important roles in development. There are precedents for stage-specific transcripts in $P$. infestans without clear functions, based on the absence of open reading frames (Avrova et al. 2007).
Groups with highest expression in zoospore stages.

Groups I and J represent some of the largest clusters in Figure 4, with 1,045 and 1,122 members, respectively. These typically have little expression in hyphae, modest levels in sporulation,

Table 2. (continued from preceding page)

\begin{tabular}{|c|c|c|c|c|c|c|c|c|}
\hline \multirow[b]{2}{*}{ Group, rank $^{b}$} & \multirow[b]{2}{*}{ Gene } & \multicolumn{5}{|c|}{ Normalized expression $^{a}$} & \multirow[b]{2}{*}{ Best BLASTX match ${ }^{\mathrm{c}}$} & \multirow[b]{2}{*}{$E$ value $^{\text {d }}$} \\
\hline & & HY & SP & $\mathbf{C L}$ & $\mathbf{Z O}$ & GC & & \\
\hline \multicolumn{9}{|l|}{ Group F (66\%) } \\
\hline 1 & Pi006549 & 26.0 & 7.7 & 0.6 & 0.0 & 0.9 & $\beta-1,3-G l u c a n$ synthase, Nicotiana alata AF304372 & $1 e-39$ \\
\hline 3 & Pi000008 & 36.1 & 10.9 & 3.0 & 0.0 & 0.1 & Proteasome $\alpha$ subunit, $O$. japonica BAA95822 & $2 \mathrm{e}-75$ \\
\hline 4 & $\mathrm{Pi} 015827$ & 13.0 & 11.4 & 2.2 & 0.0 & 0.8 & Protein phosphatase PP2A, Aspergillus oryzae BAE60375 & 0.0 \\
\hline 5 & Pi001582 & 12.3 & 5.3 & 2.5 & 0.0 & 0.1 & Chaperonin t-complex $\beta$ subunit, Trypanosoma brucei EAN77071 & 0.0 \\
\hline 6 & $\mathrm{Pi} 003860$ & 9.5 & 6.0 & 1.1 & 0.0 & 0.0 & Nucleoside diphosphate sugar epimerase, O. sativa EAY93981 & $6 e-38$ \\
\hline 7 & Pi004413 & 9.0 & 1.7 & 0.5 & 0.0 & 0.0 & $\beta$-Glucosidase, Polygonum tinctorium AAM96988 & $1 \mathrm{e}-83$ \\
\hline 9 & $\mathrm{Pi} 000046$ & 8.9 & 3.2 & 0.0 & 0.0 & 0.0 & Aspartic protease, Leptosphaeria maculans AAM81358 & $7 e-72$ \\
\hline 10 & Pi006303 & 8.8 & 4.1 & 0.1 & 0.0 & 0.4 & Chloroquine transporter, $D$. discoideum AAG31811 & $6 e-22$ \\
\hline 11 & Pi006652 & 8.6 & 2.7 & 0.0 & 0.0 & 0.4 & Esterase/lipase, Rhodococcus sp. AAG31811 & $6 e-46$ \\
\hline 12 & $\mathrm{Pi} 003740$ & 8.3 & 1.6 & 0.0 & 0.0 & 0.0 & Lysine-ketoglutarate reductase, A. thaliana AAB96826 & 0.0 \\
\hline 13 & $\mathrm{Pi} 004169$ & 7.7 & 2.0 & 0.1 & 0.0 & 0.1 & 26S proteasome regulatory subunit, X. laevis AAH79690 & $1 \mathrm{e}-85$ \\
\hline 15 & $\mathrm{Pi} 004346$ & 7.5 & 1.9 & 1.2 & 0.0 & 0.0 & Oligopeptidase b, T. brucei EAN80234 & 0.0 \\
\hline 17 & $\mathrm{Pi} 004355$ & 7.2 & 3.1 & 0.6 & 0.0 & 0.0 & Chaperonin t-complex epsilon subunit, Gallus gallus NP_001012 & 0.0 \\
\hline 22 & Pi008808 & 6.8 & 1.6 & 0.0 & 0.0 & 0.0 & Leucine aminopeptidase, A. aegypti EAT47208 & $4 e-97$ \\
\hline 23 & Pi000740 & 46.6 & 12.5 & 3.6 & 0.1 & 1.7 & Malate dehydrogenase, $X$. laevis AAX19496 & $1 \mathrm{e}-102$ \\
\hline 27 & Pi001323 & 6.0 & 2.6 & 0.1 & 0.0 & 1.9 & Prefoldin chaperone subunit 5, D. rerio XP_687759 & $7 e-26$ \\
\hline 28 & Pi009846 & 5.8 & 2.1 & 0.0 & 0.0 & 0.0 & Major facilitator superfamily protein, T. thermophila EAR94487 & $6 e-36$ \\
\hline 29 & $\mathrm{Pi} 012984$ & 5.5 & 2.2 & 0.0 & 0.0 & 0.4 & Prostaglandin synthase, $A$. thaliana AAN65132 & $1 e-52$ \\
\hline 30 & PI015921 & 5.2 & 2.3 & 1.3 & 0.0 & 1.4 & GTP-binding protein, Apis mellifera XP_392284 & $8 \mathrm{e}-84$ \\
\hline 31 & PI001414 & 5.2 & 2.9 & 1.0 & 0.0 & 0.1 & Centractin, B. taurus XP_592209 & 0.0 \\
\hline \multicolumn{9}{|l|}{ Group G $(24 \%)$} \\
\hline 2 & Pi005728 & 0.0 & 29.8 & 0.3 & 0.1 & 2.7 & Elicitin-like protein, P. ramorum ABB55956 & $2 \mathrm{e}-30$ \\
\hline 7 & Pi003022 & 0.0 & 10 & 1.0 & 0.0 & 0.1 & Loricrin-like protein, $O$. sativa $\mathrm{BAD} 45822$ & $1 \mathrm{e}-22$ \\
\hline 21 & $\mathrm{Pi} 006300$ & 0.0 & 7.4 & 0.2 & 0.0 & 0.1 & Ankryn repeat protein, D. discoideum AAS38712 & $2 \mathrm{e}-17$ \\
\hline 23 & $\mathrm{Pi} 015565$ & 0.1 & 32.1 & 3.8 & 1.3 & 3.6 & Serine/threonine protein kinase, C. albicans XP_720743 & $1 \mathrm{e}-20$ \\
\hline 25 & $\mathrm{Pi} 001305$ & 0.0 & 2.5 & 0.0 & 0.0 & 0.0 & Caltractin-like protein, A. thaliana AAM63702 & $1 \mathrm{e}-13$ \\
\hline 28 & $\mathrm{Pi} 012681$ & 0.0 & 1.9 & 0.1 & 0.0 & 0.0 & Tetratricopeptide repeat protein, $H$. sapiens NP_938051 & 0.0 \\
\hline 29 & $\mathrm{Pi} 003234$ & 0.0 & 1.7 & 0.1 & 0.0 & 0.0 & Serine/threonine protein kinase, Acanthamoeba polyphaga Q7T6X2 & $7 e-12$ \\
\hline 31 & $\mathrm{Pi} 012315$ & 0.0 & 6.6 & 0.2 & 0.0 & 0.3 & Leucine-rich repeat protein, $M$. truncatula ABE93295 & $4 \mathrm{e}-12$ \\
\hline 32 & $\mathrm{Pi} 008762$ & 0.0 & 1.4 & 0.0 & 0.0 & 0.0 & ABC transporter, $O$. sativa $\mathrm{NP}_{2} 908650$ & $4 \mathrm{e}-121$ \\
\hline 41 & Pi013200 & 0.0 & 1.2 & 0.0 & 0.0 & 0.0 & GTP-binding protein, Trichodesmium erythraeum YP_723316 & $4 \mathrm{e}-15$ \\
\hline 42 & $\mathrm{Pi} 002823$ & 0.1 & 8.0 & 0.7 & 0.3 & 0.2 & Cysteine dioxygenase, Caenorhabditis briggsae CAE7̄3080 & $2 \mathrm{e}-22$ \\
\hline 43 & $\mathrm{Pi} 003747$ & 0.0 & 3.0 & 0.0 & 0.0 & 0.0 & Retinoblastoma-binding protein, S. purpuratus XP_783715 & 0.0 \\
\hline 48 & Pi002736 & 0.0 & 1.1 & 0.0 & 0.3 & 0.0 & Ubiquitin ligase F-box protein, A. fumigatus XP_750347 & $4 e-24$ \\
\hline 66 & $\mathrm{Pi} 007247$ & 0.0 & 0.6 & 0.0 & 0.0 & 0.0 & Clusterin associated protein, $S$. purpuratus XP_781652 & $7 e-68$ \\
\hline 74 & $\mathrm{Pi005523}$ & 0.0 & 1.8 & 0.0 & 0.0 & 0.0 & Leucine-rich repeat protein, $O$. sativa BAD19701 & $6 e-14$ \\
\hline 76 & $\mathrm{Pi} 004506$ & 0.0 & 1.1 & 0.0 & 0.4 & 0.1 & Adenosyl-binding signaling protein, O. sativa ABA95929 & $2 \mathrm{e}-21$ \\
\hline 77 & $\mathrm{Pi} 008690$ & 0.1 & 3.1 & 0.1 & 0.0 & 0.7 & HMG-box DNA binding protein, $R$. norvegicus XP_001062 & $1 \mathrm{e}-11$ \\
\hline 79 & Pi003828 & 0.0 & 0.4 & 0.1 & 0.0 & 0.0 & WD40 domain protein, Canis familiaris XP_53261 & 0.0 \\
\hline 83 & $\mathrm{Pi} 013922$ & 0.0 & 0.4 & 0.0 & 0.0 & 0.0 & PDR-like ABC transporter, A. thaliana AAD 39650 & $1 \mathrm{e}-170$ \\
\hline 85 & Pi011572 & 0.0 & 0.4 & 0.0 & 0.0 & 0.0 & WD40 domain protein, $S$. purpuratus XP_782303 & $2 \mathrm{e}-78$ \\
\hline \multicolumn{9}{|l|}{ Group H (21\%) } \\
\hline 2 & Pi005938 & 0.0 & 25.3 & 27.8 & 18.9 & 1.1 & Phosphatidylinositol kinase-ubiquitin, M. truncatula ABE80823 & $1 e-36$ \\
\hline 3 & $\mathrm{Pi} 010255$ & 0.0 & 17.1 & 29.4 & 7.6 & 1.5 & Adaptin clathrin regulator, A. mellifera XP_394621 & 0.0 \\
\hline 8 & $\mathrm{Pi} 006740$ & 0.0 & 12.0 & 9.6 & 10.9 & 3.1 & Sugar oxidoreductase, Schizosaccharomyces pombe CAA20147 & $6 e-60$ \\
\hline 16 & Pi003864 & 0.1 & 105.2 & 131.1 & 71.6 & 13.0 & cdc14 phosphatase, $P$. infestans AAP38170 & 0.0 \\
\hline 17 & Pi003671 & 0.0 & 6.8 & 7.2 & 2.5 & 0.0 & Dynein heavy chain family protein, T. thermophila EAS01943 & $2 \mathrm{e}-30$ \\
\hline 18 & $\mathrm{Pi004848}$ & 0.0 & 9.4 & 4.5 & 6.9 & 1.6 & Puf-like RNA-binding protein, $P$. infestans AAN37689 & 0.0 \\
\hline 27 & $\mathrm{Pi} 007288$ & 0.0 & 17.0 & 21.8 & 14.6 & 3.9 & Acid phosphatase, $R$. norvegicus AAH90036 & $3 e-27$ \\
\hline 43 & Pi000986 & 0.0 & 3.4 & 3.0 & 2.2 & 0.4 & Flagellar root SF-assemblin, $P$. yoelii XP_726111 & $1 \mathrm{e}-16$ \\
\hline 51 & $\mathrm{Pi} 007670$ & 0.0 & 3.0 & 2.3 & 0.0 & 0.0 & DDE endonuclease/transposase, Chaetomium globosum EAQ83148 & $1 \mathrm{e}-17$ \\
\hline 52 & Pi005001 & 0.0 & 1.9 & 3.1 & 0.5 & 0.0 & $\alpha$ Tubulin suppressor, T. thermophila XP_001031 & $6 e-22$ \\
\hline 57 & $\mathrm{Pi001314}$ & 0.0 & 6.0 & 2.9 & 0.0 & 0.1 & RAS oncogene family-like, S. purpuratus XP_001178 & $1 \mathrm{e}-32$ \\
\hline 64 & Pi001137 & 0.0 & 2.8 & 1.2 & 0.0 & 0.0 & 28-kDa inner dynein arm light chain, S. purpuratus Q26630 & $3 e-66$ \\
\hline 67 & $\mathrm{Pi} 007315$ & 0.0 & 1.9 & 1.9 & 0.0 & 0.1 & Flagellar protein, SPEF-like, C. reinhardtii AAX56300 & $3 e-24$ \\
\hline 82 & Pi002279 & 0.1 & 8.3 & 8.5 & 6.3 & 0.8 & Dynein heavy polypeptide 1, C. familiaris XP_53380 & $4 \mathrm{e}-40$ \\
\hline 84 & $\mathrm{Pi005726}$ & 0.0 & 3.5 & 4.2 & 0.7 & 0.0 & Dynein regulatory complex, C. reinhardtii AAP57169 & e-101 \\
\hline 89 & Pi000618 & 0.0 & 8.1 & 5.5 & 3.2 & 0.6 & Deflagellation-inducible protein, C. reinhardtii AAD27849 & $1 \mathrm{e}-32$ \\
\hline 94 & $\mathrm{Pi} 004591$ & 0.0 & 1.2 & 1.4 & 0.3 & 0.0 & Kinesin motor protein, Tribolium castaneum XP_972069 & $9 \mathrm{e}-71$ \\
\hline 95 & Pi002480 & 0.0 & 1.6 & 1.0 & 0.0 & 0.0 & Nucleoside diphosphate kinase, Tetraodon nigroviridis CAG05664 & $2 \mathrm{e}-36$ \\
\hline 96 & Pi015853 & 0.0 & 3.1 & 3.3 & 1.5 & 0.4 & 8-kDa dynein light chain, T. brucei XP_828164 & $3 e-16$ \\
\hline \multirow[t]{2}{*}{98} & Pi011401 & 0.0 & 1.6 & 0.7 & 0.0 & 0.0 & Cell adhesion protein, Thalassiosira weissflogii AAD41094 & $6 e-17$ \\
\hline & & & & & & & & (continued on next page \\
\hline
\end{tabular}


peak levels during zoosporogenesis (group I) or in swimming zoospores (group J), and low levels in germinated cysts.

Notable in group I is the high frequency of regulatory proteins, with 8 of the 20 top genes matching protein kinases. These include several regulated by cyclic nucleotides or calcium, the latter being a well-known regulator of oomycete spores (Warburton and Deacon 1998), and a G-protein $\alpha$ subunit shown previously to be required for zoospore swimming (Latijnhouwers et al. 2004). Also represented are proteins involved in proteolysis, including two peptidases and a ubiquitin ligase. This may imply that much protein turnover occurs during cleavage, although such a role for the ubiquitin ligase may

Table 2. (continued from preceding page)

\begin{tabular}{|c|c|c|c|c|c|c|c|c|}
\hline \multirow[b]{2}{*}{ Group, rank ${ }^{b}$} & \multirow[b]{2}{*}{ Gene } & \multicolumn{5}{|c|}{ Normalized expression $^{a}$} & \multirow[b]{2}{*}{ Best BLASTX match ${ }^{c}$} & \multirow[b]{2}{*}{$E$ value $^{\mathrm{d}}$} \\
\hline & & HY & SP & $\mathbf{C L}$ & $\mathbf{Z O}$ & GC & & \\
\hline \multicolumn{9}{|l|}{ Group I (18\%) } \\
\hline 3 & $\mathrm{Pi002213}$ & 0.0 & 9.4 & 115.8 & 76.4 & 1 & Nuclear LIM-interactor (NIF) phosphatase, H. sapiens AAD09331 & $5 e-51$ \\
\hline 4 & $\mathrm{Pi002536}$ & 0.0 & 8.7 & 114 & 6.2 & 0.4 & 1,3- $\beta$-Glucanosyltransferase, $C$. glabrata CAG58677 & $2 \mathrm{e}-32$ \\
\hline 7 & $\mathrm{Pi} 004660$ & 0.0 & 56.2 & 99.7 & 27.8 & 2.1 & Vacuolar protein sorting factor 26, D. rerio AAH75958 & $4 \mathrm{e}-48$ \\
\hline 10 & $\mathrm{Pi} 012270$ & 0.0 & 0.5 & 89.2 & 3.7 & 0.0 & Extracellular dioxygenase, A. fumigatus XP_747769 & $5 e-68$ \\
\hline 19 & $\mathrm{Pi} 005723$ & 0.0 & 5.1 & 58.2 & 34.6 & 1.2 & Ascorbate-dependent monooxygenase, Aplysia brasiliana AAS92605 & $1 \mathrm{e}-13$ \\
\hline 31 & $\mathrm{Pi004077}$ & 0.0 & 0.2 & 37.6 & 26.3 & 1.6 & Calmodulin-like domain protein kinase, Eimeria tenella ABA60893 & $1 e-37$ \\
\hline 45 & $\mathrm{Pi003506}$ & 0.0 & 1.4 & 28.6 & 2.5 & 0.0 & Serine/threonine protein kinase, A. mellifera XP_001123105 & $1 e-33$ \\
\hline 51 & $\mathrm{Pi} 000068$ & 0.0 & 1.7 & 24.1 & 1.9 & 0.0 & cAMP regulated protein kinase, $T$. nigroviridis CAF98611 & $1 e-31$ \\
\hline 65 & $\mathrm{Pi} 013754$ & 0.0 & 1.2 & 19.0 & 12.1 & 2.8 & Serine/threonine protein kinase, $O$. sativa AAO72550 & $2 \mathrm{e}-19$ \\
\hline 71 & $\mathrm{Pi003370}$ & 0.0 & 1.9 & 17.6 & 1.7 & 0.5 & Cation-transporting ATPase, C. briggsae CAE66142 & 0.0 \\
\hline 72 & Pi003366 & 0.0 & 0.6 & 16.3 & 1.8 & 0.1 & Calmodulin-domain protein kinase, T. gondii AAG53993 & $2 e-57$ \\
\hline 80 & $\mathrm{Pi} 015784$ & 0.0 & 6.2 & 14.8 & 1.8 & 0.4 & Mitogen-activated protein kinase, C. reinhardtii BAB18271 & $2 \mathrm{e}-112$ \\
\hline 82 & Pi001698 & 0.0 & 4.4 & 50.4 & 1.9 & 0.5 & cAMP-dependent protein kinase, T. gondii AAX55640 & $1 \mathrm{e}-104$ \\
\hline 83 & $\mathrm{Pi003034}$ & 0.0 & 6.4 & 54.3 & 9.1 & 1.3 & Serine/threonine protein kinase, T. thermophila EAR87956 & $1 \mathrm{e}-77$ \\
\hline 88 & $\mathrm{Pi} 014774$ & 0.0 & 0.3 & 12.7 & 0.5 & 0.0 & Metallopeptidase, C. familiaris XP_851508 & $2 \mathrm{e}-54$ \\
\hline 90 & Pi004311 & 0.0 & 3.6 & 23.8 & 2.7 & 0.1 & Acidic dipeptidase, M. musculus XP_984088 & $1 e-55$ \\
\hline 100 & $\mathrm{Pi000084}$ & 0.1 & 5.4 & 49 & 9.4 & 0.7 & G-protein $\alpha$ subunit, $P$. infestans AAL11436 & 0.0 \\
\hline 104 & Pi011657 & 0.0 & 1.2 & 8.2 & 0.9 & 0.1 & E3 ubiquitin ligase, Lotus japonica BAF38781 & $3 e-83$ \\
\hline 130 & $\mathrm{Pi} 011670$ & 0.0 & 0.1 & 7.2 & 1.0 & 0.1 & Sugar phosphatase, HAD family, A. thaliana CAB75787 & $63-52$ \\
\hline 134 & $\mathrm{Pi001429}$ & 0.0 & 3.9 & 6.8 & 2.6 & 0.1 & Rab23 vesicular trafficking GTPase, D. melanogaster AAF51970 & $2 \mathrm{e}-33$ \\
\hline \multicolumn{9}{|l|}{ Group J (14\%) } \\
\hline 4 & Pi002501 & 0.0 & 0.0 & 1.1 & 70.9 & 4.0 & Elicitin-like protein, P. infestans ABB55936 & $3 e-15$ \\
\hline 5 & Pi004691 & 0.0 & 0.0 & 2.4 & 53.2 & 2.8 & Endoglucanase, Hahella chejuensis YP_434834 & $6 e-15$ \\
\hline 8 & $\mathrm{Pi006566}$ & 0.0 & 0.0 & 6.2 & 35.6 & 9.4 & $\beta-1,3$-Endoglucanase, A. fumigatus AAF 13033 & $2 \mathrm{e}-70$ \\
\hline 13 & Pi000066 & 0.1 & 0.5 & 94.5 & 145.6 & 57.7 & Cellulase, Thermobispora bispora $\mathrm{P} 26414$ & $8 \mathrm{e}-19$ \\
\hline 33 & $\mathrm{Pi002441}$ & 0.0 & 0.1 & 1.5 & 31.7 & 5.3 & Cutinase, Kineococcus radiotolerans EAM73881 & $2 \mathrm{e}-44$ \\
\hline 65 & Pi003052 & 0.0 & 0.0 & 1.7 & 4.4 & 0.0 & 3-Isopropylmalate dehydrogenase, Francisella tularensis ABK88970 & $2 \mathrm{e}-102$ \\
\hline 70 & Pi009653 & 0.0 & 0.0 & 2.2 & 3.8 & 0.0 & ABC transporter, T. thermophila XP_001007130 & $1 e-36$ \\
\hline 75 & $\mathrm{Pi0} 15212$ & 0.1 & 0.8 & 18.9 & 45.2 & 3.5 & Ribonucleotide reductase, Carassius carassius AAY82584 & $9 e-91$ \\
\hline 78 & $\mathrm{Pi0} 13446$ & 0.0 & 0.0 & 1.5 & 3.2 & 0.6 & ATP-dependent DNA ligase, X. laevis AAH74385 & $3 e-98$ \\
\hline 85 & Pi003184 & 0.1 & 0.4 & 3.0 & 16.7 & 1.7 & Mitochondrial carrier protein, $M$. grisea XP_362038 & $1 e-49$ \\
\hline 88 & $\mathrm{Pi} 005850$ & 0.1 & 2.0 & 18.3 & 34.9 & 5.0 & Quinolinate synthase, A. thaliana AAM6277̄6 & $6 e-129$ \\
\hline 90 & $\mathrm{Pi} 007838$ & 0.1 & 0.0 & 11.0 & 33.6 & 9.1 & FAD/FMN dehydrogenase, Gibberella zeae EAA71564 & $3 e-64$ \\
\hline 92 & $\mathrm{Pi003883}$ & 0.0 & 0.8 & 0.8 & 2.3 & 1.3 & Serine/threonine protein kinase, $O$. sativa XP_472963 & $2 \mathrm{e}-101$ \\
\hline 100 & $\mathrm{Pi008298}$ & 0.0 & 0.0 & 1.4 & 10.0 & 0.7 & ß-Glucosidase Cytophaga hutchinsonii ZP_00308266 & $5 e-17$ \\
\hline 104 & $\mathrm{Pi003247}$ & 0.3 & 0.0 & 12.2 & 57.5 & 6.6 & Long-chain acyl-CoA synthetase, X. laevis AAH43850 & 0.0 \\
\hline 110 & $\mathrm{Pi} 003868$ & 0.3 & 0.7 & 37.6 & 67.4 & 15 & Oxalate/formate MFS antiporter, D. discoideum EAL70815 & $3 e-68$ \\
\hline 113 & $\mathrm{Pi003507}$ & 0.2 & 0.0 & 2.6 & 10.3 & 2.0 & Mitochondrial carrier protein, C. briggsae CAE60856 & $1 e-41$ \\
\hline 121 & Pi006411 & 0.0 & 0.0 & 0.7 & 3.2 & 0.4 & DEAD-box helicase, D. discoideum XP_640452 & $9 e-90$ \\
\hline 133 & $\mathrm{Pi003465}$ & 0.2 & 0.1 & 6.0 & 21.4 & 3.5 & Sodium:dicarboxylate symporter, Solibacter usitatus EAM57970 & $1 e-79$ \\
\hline 140 & Pi002732 & 0.4 & 1.0 & 19.0 & 42.3 & 11.1 & Tyrosine kinase, D. discoideum XP_636072 & $2 \mathrm{e}-29$ \\
\hline \multicolumn{9}{|l|}{ Group K $(35 \%)$} \\
\hline 2 & $\mathrm{Pi} 001770$ & 0.0 & 0.0 & 0.4 & 38.2 & 56.1 & Necrosis \& ethylene inducing protein, Bacillus halodurans NP_241261 & $9 e-69$ \\
\hline 6 & $\mathrm{Pi000795}$ & 0.2 & 0.0 & 2.7 & 70.1 & 73.6 & Carbonic anhydrase, Lyngbya sp. EAW37375 & $4 \mathrm{e}-24$ \\
\hline 19 & $\mathrm{Pi} 015763$ & 2.4 & 1.1 & 12.1 & 108.5 & 103.4 & ABC transporter, Aspergillus fumigatus XP_755735 & $3 e-80$ \\
\hline 21 & $\mathrm{Pi001624}$ & 0.2 & 0.0 & 0.8 & 4.7 & 7.6 & Transmembrane amino acid transporter, $U$. maydis UM02159 & $5 e-10$ \\
\hline 22 & $\mathrm{Pi} 015265$ & 0.1 & 0.0 & 0.0 & 0.9 & 1.6 & Endoglucanase, Aspergillus clavatus XP_001269687 & $1 e-55$ \\
\hline 26 & Pi002491 & 3.3 & 0.0 & 4.2 & 64.1 & 69.5 & Cellulase, Irpex lacteus BAA76365 & 0.0 \\
\hline 27 & Pi003533 & 1.5 & 0.0 & 2.8 & 29.0 & 28.5 & Cellulase, Humicola grisea BAA09785 & 0.0 \\
\hline 29 & Pi006346 & 0.8 & 1.8 & 2.2 & 13.0 & 13.6 & Ascorbate-dependent monooxygenase, Aplysia californica AAY42041 & $2 \mathrm{e}-13$ \\
\hline 30 & $\mathrm{Pi} 002310$ & 2.1 & 2.7 & 3.3 & 17.6 & 26.7 & $\beta$-Glucosidase, $C$. hutchinsonii YP_678873 & $2 \mathrm{e}-125$ \\
\hline 33 & $\mathrm{Pi} 005156$ & 0.9 & 1.5 & 1.5 & 6.7 & 7.3 & 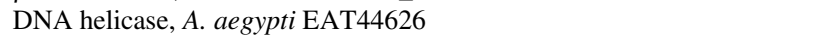 & $1 \mathrm{e}-92$ \\
\hline 40 & $\mathrm{Pi0} 15252$ & 1.0 & 0.2 & 0.9 & 6.3 & 7.8 & Histone H4, Solanum chacoense AAQ24536 & $2 \mathrm{e}-36$ \\
\hline 43 & Pi014900 & 0.6 & 1.2 & 1.1 & 3.8 & 4.9 & Threonine dehydratase, A. mellifera XP_624902 & $1 \mathrm{e}-124$ \\
\hline 46 & Pi007381 & 0.9 & 0.3 & 0.7 & 5.4 & 6.9 & Retroelement polyprotein, S. purpuratus XP_786907 & $7 e-21$ \\
\hline 49 & Pi003261 & 3.7 & 1.3 & 2.2 & 25.3 & 20.7 & Elicitin-like protein, $P$. infestans ABB55933 & $6 e-56$ \\
\hline 51 & $\mathrm{Pi005237}$ & 0.1 & 0.0 & 0.0 & 0.4 & 0.7 & Proteasome subunit $\beta, D$. rerio NP_571227 & $1 e-60$ \\
\hline 52 & Pi012034 & 1.4 & 3.1 & 2.3 & 5.6 & 7.0 & Kinesin motor protein C3, C. familiaris XP_544385 & $2 \mathrm{e}-78$ \\
\hline 53 & Pi013746 & 1.9 & 1.6 & 3.0 & 9.1 & 9.2 & WD40-containing tyrosine kinase, D. discoideum EAL65683 & $1 e-37$ \\
\hline 54 & Pi001798 & 2.0 & 4.9 & 4.2 & 7.6 & 9.6 & Secretory vesicle SNARE protein, Anopheles gambiae EAA06868 & $3 e-08$ \\
\hline 55 & Pi002141 & 3.0 & 3.4 & 3.1 & 11.3 & 14.0 & FtsZ inhibiting protein MinC, Chromobacterium violaceum Q7NSP5 & 1e-17 \\
\hline 56 & Pi009901 & 0.9 & 1.0 & 1.1 & 3.8 & 4.6 & Thioesterase, T. brucei XP_844018 & $2 \mathrm{e}-31$ \\
\hline
\end{tabular}


be unlikely because, as described later, mRNAs for the proteasome fall in spores. An alternative explanation for induction of the ubiquitin ligase is related to the role of such proteins in regulating the movement of vesicles (Hicke and Dunn 2003). Interestingly, also in group I are a vesicular trafficking ATPase and a vacuolar sorting protein. Such proteins may be important in releasing stored glucans or proteins during encystment, or delivering material to the cyst germ tube.

Group $\mathbf{J}$ also includes several regulators such as protein kinases. However, it is distinguished from group I by an abundance of glycosyl hydrolases such as cutinase. Therefore, it appears that many transcripts required for host invasion begin to accumulate during zoosporogenesis. Group $\mathbf{J}$ also differs from group I by the predominance of genes involved in general metabolism and energy generation, including three transporters for citric acid cycle intermediates and quinolinate synthase, which acts in NAD biosynthesis. Two proteins required for DNA synthesis, ribonucleotide reductase and DNA ligase, also are induced at this stage, possibly in anticipation of the resumption of chromosomal replication after cyst germination.

\section{Genes predominantly expressed in germinated cysts.}

Groups B and K, with 357 and 457 members, respectively, are largely specific to the stage containing germinated cysts with appressoria, although group $\mathrm{K}$ genes start being induced in the preceding zoospore stage. As in many other groups, protein kinases are well represented, with four listed in Table 2. Also present are genes predicted to encode structural proteins; most do not appear in Table 2 because they lack matches against other proteins, except for Pi005101, which encodes a low-complexity 838 -amino-acid protein (25\% ser, $11 \%$ thr) that weakly matches a Candida adhesion protein.

Many genes in groups B and $\mathrm{K}$ have potential roles in plant pathogenesis. Several may guard against plant defense toxicants such as reactive oxygen species and phytoalexins; these include glutathione $S$-transferase and catalase/peroxidase in group B, and an ABC transporter in group $\mathrm{K}$. Also represented in both groups are cell-wall-degrading enzymes (annotated as cutinase, xylanase, cellulase, $\beta$-glucosidase, and endoglucanase), although roles for some in the biogenesis of the P. infestans wall is also possible because both oomycete and plant walls are rich in cellulose and $\beta-1,3$ and $\beta-1,6$ glucans (Bartnicki-Garcia and Wang 1983). Group K also includes a plant cell necrosis and ethylene-inducing protein, NPP1. Work in $P$. sojae indicated that the NPP1-encoding PsojNIP gene is upregulated late in infection, during the transition from biotrophic to necrotic growth (Qutob et al. 2002); however, some genes in the family apparently are expressed at an earlier stage, as shown earlier for PiNPP1.2 and PiNPP1.3 of P. infestans (Kanneganti et al. 2006). Many of the genes described above encode proteins that are secreted or membrane-associated, which also suggest roles in the plant interaction. Besides those mentioned above, such genes include Pi010868, which encodes a membrane protein that matches an Aspergillus fumigatus zinc ion transporter, and Pi001624, which encodes a membrane protein resembling a Ustilago maydis amino acid transporter.

Another type of secreted factor represented by several genes in group B are RXLR proteins, which are effectors that enter plant cells during infection (Birch et al. 2006). Recently, it was demonstrated that AVR3a of P. infestans is translocated by virtue of its RXLR motif from haustoria into host cells (Whisson et al. 2007). RXLR-encoding genes, including those indicated in group B, also are known to be upregulated during the biotrophic phase of the interaction with potato (Whisson et al. 2007). Combined with the observations described in the preceding paragraph, such results indicate that genes required for host colonization, including participants in the biotrophic haustoria-forming stages, are upregulated in the prior stages of the infection cycle (i.e., germinating cysts). The biotrophic stages of infection are hard to study using microarrays due to the low amount of pathogen biomass; therefore, examining preinfection stages such as germinating cysts is a useful alternative for gaining insight into the earliest transcriptional changes required for establishing the interaction with the host.

\section{Genes with elevated expression in hyphae.}

Four groups have higher levels in hyphal stages than spores: groups C and D, with 575 and 337 members, respectively, showing much higher levels in hyphae and germinating cysts than any spore stage; and E and F, with 446 and 1,064 members, respectively, having high expression in hyphae but not germinated cysts.

Abundant in these groups are participants in metabolism and nutrient uptake. For example, listed in Table 2 are six sugar or amino acid transporters, a glycolytic enzyme, and various dehydrogenases (oxidoreductases). The downregulation of these genes in sporangia and zoospores relative to the filamentous stages is consistent with the generation of energy for spores from a narrow range of stored nutrients, particularly lipids and mycolaminarin (Bartnicki-Garcia and Wang 1983). This is in contrast to the situation in hyphae where many compounds likely are transported from the plant and used as metabolic substrates.

Many other metabolic enzymes are represented in the four groups, but divining their roles is challenging because they often play multiple functions or catalyze bidirectional reactions. For example, malate dehydrogenase (group F) converts malate into oxaloacetate, which can be used in the citric acid cycle to generate NADH or as a substrate for phosphoenolpyruvate carboxykinase during gluconeogenesis. Similarly, group E includes pyruvate phosphate dikinase, which interchangeably converts phosphoenolpyruvate and pyrophosphate (PPi) to pyruvate and ATP, thereby linking glycolysis, lipid oxidation, and PPi-producing pathways. Whether this hyphal-specific pyruvate phosphate dikinase preferentially acts in one direction or the other cannot be surmised, but its presence in Phytophthora spp. was taken as evidence of the importance of PPi-based metabolism (Marshall et al. 2001). Another enzyme involved in PPi metabolism, ATP sulfurylase, is also in group E. This reversibly transfers sulfate to the adenine monophosphate component of ATP to form adenosine 5-phosphosulfate and PPi. By catalyzing the initial step of sulfate activation, ATP sulfurylase is necessary for methionine and cysteine biosynthesis, which is consistent with the higher activity of these pathways in hyphae versus spores (Grenville-Briggs et al. 2005).

Many participants in protein turnover are also represented in the groups showing higher mRNA levels in hyphae. For example, within the top 20 genes of the groups are five proteases, three signalosome (proteasome) subunits, and three chaperonins. Their downregulation in spores suggests that protein breakdown is not a major source of energy or building blocks for new protein.

Groups C and D also encode several potential pathogenicity factors or effectors, which would be expected to be produced in germinating cysts or hyphae, at least in planta. These include a member of the RXLR family of plant effectors, a protease inhibitor, peroxidase, and three cytochrome P450 enzymes potentially used to degrade phytoalexins. Also represented are four glucanases that could contribute to host cell wall degradation. The expression of the latter are in contrast to a $\beta-1,3$-glucan synthase in group $\mathrm{F}$, and two $\beta$-glucosidases ( $\mathrm{E}$ and $\mathrm{F}$ ) whose expression is not induced in germinating cysts. It is possible that these genes may function in the synthesis of the Phytophthora cell wall. 
Groups $\mathrm{C}$ and $\mathrm{D}$, but not $\mathrm{E}$ and $\mathrm{F}$, include many regulatory proteins. These include two $m y b$-like transcription factors, three protein kinases, an RAB GTPase regulator, and a cyclin. These are attractive candidates for future studies, especially the predicted cdc2 kinase that might regulate cell cycle progression during filamentous growth. However, the presence of a retroelement-like sequence in group $\mathrm{D}$ is a reminder that having a stage-specific expression does not guarantee the biological relevance of any transcript.

\section{Genes classified by function.}

To better understand the changes occurring during development, genes involved in selected activities were analyzed. In some cases, genes associated with a particular function were expressed coordinately whereas other classes exhibited divergence. In the case of the latter, statistical clustering combined with visual inspection of the data helped categorize genes into subclasses. Notable findings are summarized below and in Figure 5; the underlying data, including descriptions of genes in each class, are presented in Supplementary Table 2.

Some classes of genes have relatively constant expression during development. These include those involved in basal transcription (41 RNA polymerase subunits and TFII proteins) and translation (118 genes encoding ribosomal proteins plus initiation and elongation factors). Their mRNA levels are slightly higher in hyphae and germinated cysts than spores, although the slight depression in spores may result from the high expression of many spore-specific genes.
Participants in DNA replication were examined by studying 20 genes encoding DNA polymerase subunits, origin recognition complex proteins, and replication licensing factors. Most show either moderate increases or decreases during spore stages (subclasses A and B). The one gene having a major change encodes licensing factor Mcm5 (Cdc46 in budding yeast; BLASTX $E=0.0$ ), for which mRNA disappears during sporulation and then reappears during cleavage (subclass $\mathrm{C}$ ). This is notable because mitosis arrests once sporangia form and does not reinitiate until approximately $6 \mathrm{~h}$ after cysts germinate (Ah Fong and Judelson 2003). Therefore, Mcm5 is implicated as a key regulator of replication in the oomycete life cycle, which more closely resembles the situation in mammalian cells, where Mcm5 protein and mRNA are induced at the G1/S boundary, than in yeast, where expression persists throughout the cell cycle (Tsuruga et al. 1997).

Genes involved in energy generation exhibit very heterogeneous patterns of expression. In the case of glycolytic enzymes, mRNA levels of 7 genes increase after sporulation (subclass A), 8 are highest in hyphae and germinated cysts (subclass B), and 12 fall after sporulation (subclass $\mathrm{C}$ ). Explanations for the divergent patterns of expression of the glycolytic genes are not necessarily obvious. For example, of the two enzymes in the preparatory phosphorylation stage of glycolysis, one is in subclass A (hexokinase) and a second is in subclass B (phosphofructokinase). Also, some enzyme activities are within more than one subclass, such as pyruvate kinase, which is encoded by six genes spread throughout subclasses A, B, and C. These
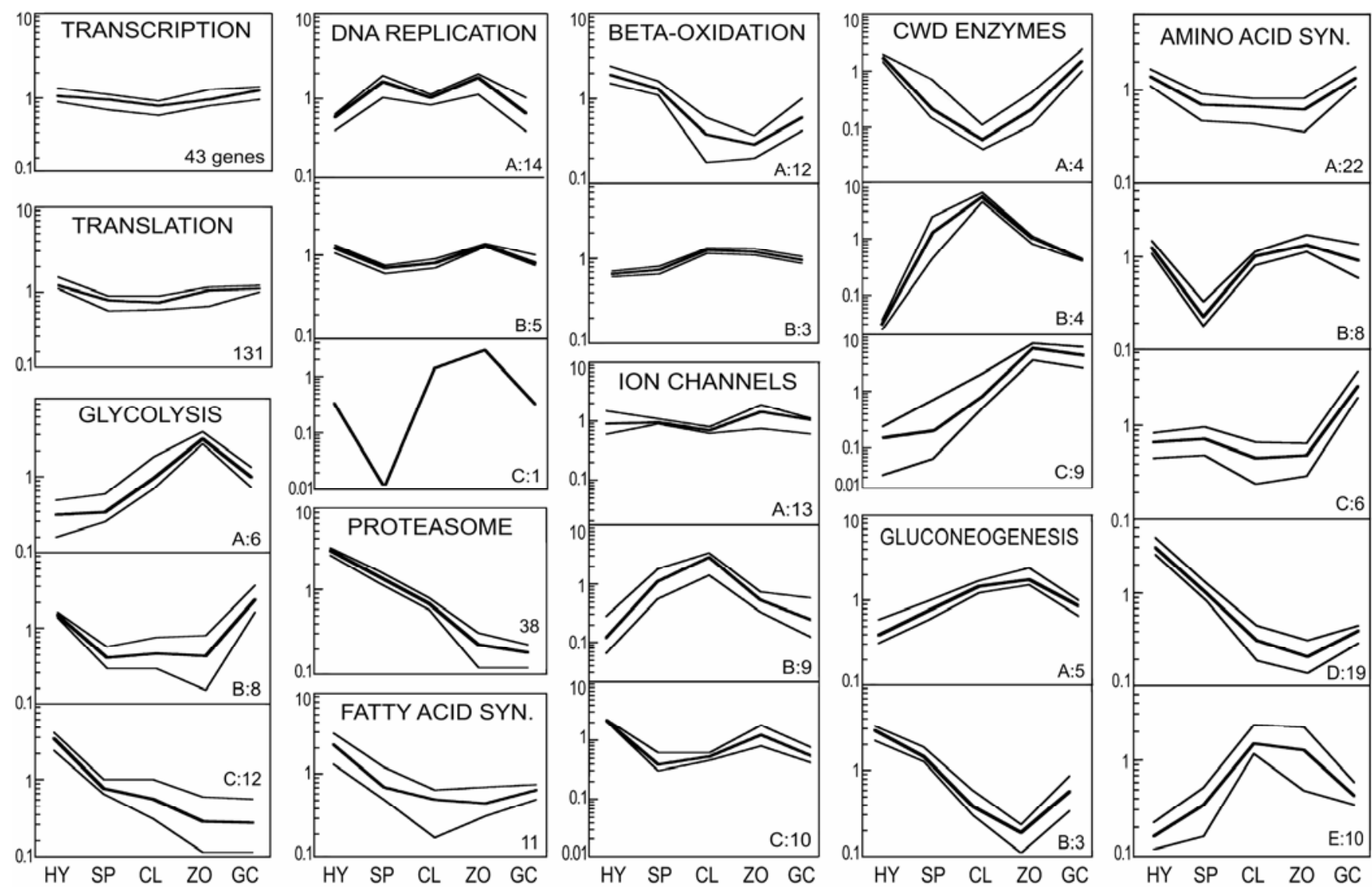

Fig. 5. Expression of genes grouped by function. Presented are the mRNA levels of genes in hyphae (HY), sporangia (SP), cleaving sporangia (CL), swimming zoospores (ZO), and germinating zoospore cysts plus appressoria (GC). The center line within each panel represents average expression, with upper and lower lines representing upper and lower quartiles. Depending on their diversity of expression patterns, genes within each functional group are presented as one class or multiple subclasses (A, B, C, and so on). The number of genes within each class or subclass is indicated in the lower right corner of each panel. In this analysis, redundancy due to two unigenes corresponding to the same gene was eliminated using comparisons to whole-genome data. For example, 43 genes encoding the basal transcription machinery matched 41 unique genes, 128 genes involved in translation matched 118 genes, and 29 encoding glycolytic enzymes matched 27 genes.

Vol. 21, No. 4, $2008 / 443$ 
may represent forms specialized for discrete activities or regulated by different effectors, because pyruvate kinase not only acts in glycolysis but also generates substrates for amino acid biosynthesis and regulates gluconeogenic flux. Having different stage-specific forms of metabolic enzymes appears to be common in $P$. infestans, based on both this microarray study and a proteomic analysis of $P$. infestans development (P. van West, personal communication).

Genes involved in gluconeogenesis show two overall patterns, with five gradually increasing through spore stages (subclass A) and three dropping upon sporulation (subclass B). As in glycolysis, the same activities appear in different subclasses, such as fructose-2,6-bisphosphatase, which is in subclasses A and $\mathrm{B}$. In contrast, three phosphoenolpyruvate carboxykinases reside only in subclass $\mathrm{A}$, and the oxaloacetate-generating enzyme pyruvate carboxylase is encoded by a single gene in subclass B. The latter, which catalyzes the first step in the gluconeogenic pathway, shows a progressive 58-fold drop from hyphae to zoospores. This could reflect a reduced need as germination proceeds for gluconeogenesis or citric acid cycle intermediates. Possibly relevant to this is the observation that mRNAs for citric acid cycle enzymes exhibit a similar reduction (68-fold) between these stages (not shown).

Fatty acid biosynthesis and $\beta$-oxidation pathways also were examined, because lipids represent a major carbon reserve in Phytophthora spp. (Bimpong 1975). Enzymes associated with fatty acid synthesis such as fatty acid synthases, acetyl-CoA carboxylases, acyl carrier proteins, and others decline coordinately after sporulation, which is consistent with a net carbon deficit in the nonassimilative spore stages. A more complex profile describes enzymes associated with $\beta$-oxidation. Most decline moderately during zoosporogenesis and recover in germinated cysts, such as the 17 genes encoding enoyl-CoA hydratase, thiolase, or acyl-CoA dehydrogenase represented by subclass A. The restoration of these enzymes in cysts may reflect the onset of starvation as mycolaminarin reserves become depleted. However, repeating a theme seen for glycolytic proteins, a thiolase and acyl-CoA dehydrogenase are also in subclass B and show little change during development. Possibly, these represent proteins with different substrate specificities.

Amino acid biosynthetic enzymes partition into five major subclasses, based on analyses of 35 enzyme activities primarily associated with the synthesis but not catabolism of amino acids. Several complex patterns of expression are shown; however, all but subclass $E$ increase in the stage containing germinated cysts with appressoria. This is consistent with earlier studies demonstrating that levels of many amino acids fall in Phytophthora zoospores but rise by the appressoria stage, along with mRNAs for certain enzymes such as methionine synthase (GrenvilleBriggs et al. 2005; Shan et al. 2004). As in glycolysis, some proteins annotated with the same function had different expression patterns, such as alanine aminotransferases (subclasses B and C). However, this was uncommon. For example, three distinct aspartate aminotransferases are all in subclass A, two cysteine desulfurases are both in subclass $\mathrm{D}$, and three cobalamin-dependent methionine synthases (EC 2.1.1.13) are in sporeinduced subclass E. However, a cobalamin-independent methionine synthase (EC 2.1.1.14) is in subclass A.

As noted in Table 2, P. infestans expresses several sporespecific ubiquitin-associated proteins. Consequently, it was of interest to examine the expression of the 38 structural or regulatory subunits of the proteasome represented on the GeneChip. Interestingly, their mRNAs decline through the sporangia to germinated cyst stages by $>10$-fold. This suggests that the proteasome is not heavily used to recycle proteins unneeded after developmental transitions, and the vacuolar pathway may be a more likely mechanism for this activity. Notably, the two spore- induced proteinases (Table 2, group I) are predicted by PSORT to be vacuolar or cytoplasmic, not secreted, and, therefore, may function in protein turnover rather than pathogenesis. Repressing the proteosome may be a useful option for preserving needed cellular components during the nutrient-limited spore stages. Considering that spores represent a period of ceased growth and mitotic dormancy, the decline in proteasome mRNAs in Phytophthora spp. is consistent with the downregulation of that body noted in starved or growth-arrested Saccharomyces spp. (Bajorek et al. 2003).

Genes encoding cell-wall-degrading enzymes required for plant colonization such as cutinases, polygalacturonases, pectate lyases, and pectinase all display major changes during development (Fig. 5, CWD). These fall into three subclasses, being either high in hyphae and germinated cysts (subclass A), induced early in the spore pathway (subclass B), or slowly increasing through all spore stages (subclass $\mathrm{C}$ ). The differential kinetics of induction between subclasses $\mathrm{B}$ and $\mathrm{C}$ might indicate their respective specializations for initial infection events versus later stages. The high level of expression of subclass A genes in hyphae may simply reflect their induction by plant wall components in the rye-sucrose media. Cellulases were not included in this analysis because their potential for acting against plant versus the Phytophthora cell walls cannot be distinguished; however, as noted earlier, mRNAs for several such proteins are induced in spores or cysts.

Both constitutive and developmentally regulated ion channel proteins were observed. Thirteen show only slight changes in mRNA abundance during development (subclass A), five drop moderately during sporulation (subclass $\mathrm{C}$ ), and nine rise dramatically in sporangia (subclass B). The latter may be particularly significant because flux measurements and inhibitor studies indicate that ions, particularly calcium, play key roles in germination and encystment (Warburton and Deacon 1998). Based on similarity to characterized proteins, all subclasses appear to include calcium, potassium, and sodium transporters.

\section{Comparison of transcription}

in planta and on laboratory media.

When considering the data described above, especially for genes involved in metabolism, it is important to remember that the tissues employed were generated on artificial media. In particular, hyphae and sporangia were from sucrose-amended rye media, which may present a nutrient profile to $P$. infestans distinct from that of plants. Thus, it is conceivable that the higher mRNA levels seen for many metabolic enzymes in hyphae compared with zoospores is an artifact. For example, high nutrient levels in the laboratory media might artificially elevate mRNAs for certain enzymes involved in glycolysis, fatty acid biosynthesis, and so on. This probably is not as serious an issue for the cleavage, zoospore, and germinated cyst stages, which were prepared in water which more closely mimics their natural environment.

To address this issue, qRT-PCR was used to examine expression levels of five metabolic genes in infected tomato, hyphae grown on rye-sucrose media, and zoospores (Fig. 6). The genes tested were selected from those exhibiting higher levels in hyphae than spore stages, representing the pathways of amino acid biosynthesis (Pi003740, saccharopine dehydrogenase), glycolysis (Pi004574, glucose-6-phosphate isomerase), fatty acid synthesis (Pi002788, fatty acid synthase), $\beta$-oxidation (Pi003501, acyl CoA-dehydrogenase), and gluconeogenesis (Pi002988, pyruvate carboxylase). In all cases, lower mRNA abundance was detected in zoospores than hyphae regardless of whether the hyphae were produced in rye-sucrose media or in planta. This is consistent with the developing consensus that sugar levels in infected plant tissue are reasonably high at least until late infec- 
tion, although the in planta status of certain nutrients such as amino acids is still controversial (Solomon et al. 2003).

\section{Constitutively expressed genes.}

Expression profiling methods, including RT-PCR and RNA blotting, require constitutively expressed genes for normalization purposes. Consequently, the data were searched for both genes used previously as controls and those exhibiting little variation between the five developmental stages. Our previous observation that actin $\mathrm{A}(a c t A)$ and elongation factor-1 $\alpha(\mathrm{EF}-1)$ are not expressed in constant proportion to each other or ribosomal RNA (Cvitanich and Judelson 2003) was supported by the GeneChip data, because actA varied up to 3.5-fold between stages and EF-1 by 5-fold. It should be noted that the genome encodes two near-identical copies of EF-1 that would not be distinguished by the GeneChips; therefore, it is possible that one is constitutive. Actin B (actB) varied up to 12-fold, cyclophilin 5.8-fold, and $\beta$-tubulin 2.1-fold. A ubiquitin-conjugating enzyme (Pi00006) varied twofold, supporting a recent finding that it is a better control than actA and EF1 (Yan and Liou 2006).

Approximately 224 genes change $<50 \%$ between the five stages $(P<0.05)$. Most common were ribosomal proteins, with 27 represented. For example, mRNA for 40S ribosome protein S3a varied by a maximum of $35 \%$ between stages, confirming a suggestion that it would be a useful control (Yan and Liou 2006). Other elements of the protein synthesis apparatus showing little change include signal recognition particle subunits and several translation initiation factors. Eight genes exhibited less than $25 \%$ variation between stages, including a Mago nashi RNA-binding protein homologue (Pi000681), a ubiquitin-protein ligase (Pi002585), an exosome ribonuclease (Pi011686), and protoporphyrinogen oxidase (Pi004155).

\section{Conclusions.}

This study provides much new insight into the biology of $P$. infestans and other oomycetes. Approximately $60 \%$ of genes were found to exhibit greater than twofold changes in mRNA levels over the life cycle, with $15 \%$ being essentially stagespecific. This reflects a striking degree of transcriptome remodeling compared with that seen in fungal plant pathogens. For example, during teliospore germination in $U$. maydis, $5.9 \%$ of genes showed greater than twofold induction (Zahiri et al. 2005) compared with $15 \%$ during zoosporogenesis in $P$. infestans. The principal reason for the higher number of regulated genes in $P$. infestans likely is related to the dramatic structural and physiological differences between zoospores and other life stages. Another explanation may be that, because Phytophthora sporangia are fully hydrated and metabolically active, specialized methods for maintaining viability and detecting cues for germination are employed that are not found in the desiccated spores made by most true fungi. It may be more appropriate to compare $P$. infestans with an organism such as the malaria parasite Plasmodium falciparum, where at least two-thirds of genes change more than twofold between its diverse life-stages (Le Roch et al. 2003).

Many of the spore-induced genes in Phytophthora infestans represent potential targets for new crop protection chemicals. Spores are attractive targets for disease control because their environmental exposure and limited nutrient reserves engender vulnerability. Many high-priority candidates for research into the fundamental biology of oomycetes also can be gleaned from the data. For example, information about genes predominantly expressed in germinated cysts with appressoria is invaluable for studying the interaction of $P$. infestans with its hosts. Appressoria, the structures formed just prior to infection, are likely to contain many transcripts required for successful penetration of the host and establishment of a compatible interaction. Future investigations of genes induced at this and the other stages naturally will include gene silencing, because developmental regulation does not guarantee function, plus proteomics and metabolomics. In particular, our present understanding of oomycete metabolism is insufficient to fully reveal the roles of many of the enzymes discussed in this article, including those with multiple forms with disparate patterns of expression. Stage-specific transcripts lacking coding potential also need examination to determine whether they are merely distractions or part of a regulatory system that, even in more-studied organisms, is just starting to be understood (Mattick 2004).

\section{MATERIALS AND METHODS}

\section{Growth conditions and RNA extractions.}

Stocks of isolate 88069, an A1 mating type strain from the Netherlands, were maintained at $18^{\circ} \mathrm{C}$ on rye A agar (Randall et al. 2005). To obtain nonsporulating hyphae for gene expression studies, $10^{3}$ sporangia were spread on a polycarbonate membrane (0.4- $\mu \mathrm{m}$ pore) laid on the surface of rye agar; hyphae were recovered after 72 to $96 \mathrm{~h}$, typically a day before asexual sporulation would occur. To isolate asexual sporangia, 10-day rye agar plates were flooded with water and rubbed with a glass rod to generate a sporangial suspension which was filtered through 50- $\mu \mathrm{m}$ nylon mesh to remove hyphal fragments. Sporangia undergoing zoosporogenesis (cleaving sporangia) were obtained by placing a sporangial suspension at $10^{\circ} \mathrm{C}$ for $60 \mathrm{~min}$; after this time, signs of cleavage were visible in approximately $70 \%$ of sporangia, although only a small amount $(<2 \%)$ had actually released zoospores. Swimming zoospores were isolated by incubating sporangia at $10^{\circ} \mathrm{C}$ for an additional 60 to $90 \mathrm{~min}$, followed by passage through $10-\mu \mathrm{m}$ mesh to remove ungerminated sporangia and pelleting at 5,000 $\times g$ for $5 \mathrm{~min}$. To obtain germinating zoospore cysts, swimming zoospores were vortexed $60 \mathrm{~s}$ to induce encystment and incubated at $10^{\circ} \mathrm{C}$ in plastic plates overnight; after this time, nearly all cysts produced germ tubes, and many also generated appressorium-like structures. Infected plant tissue was obtained by dipping detached tomato leaflets (cv. New Yorker) into zoospore suspensions, followed by incubation for 4 days at $16^{\circ} \mathrm{C}$ with a 12-h day-and-night cycle.

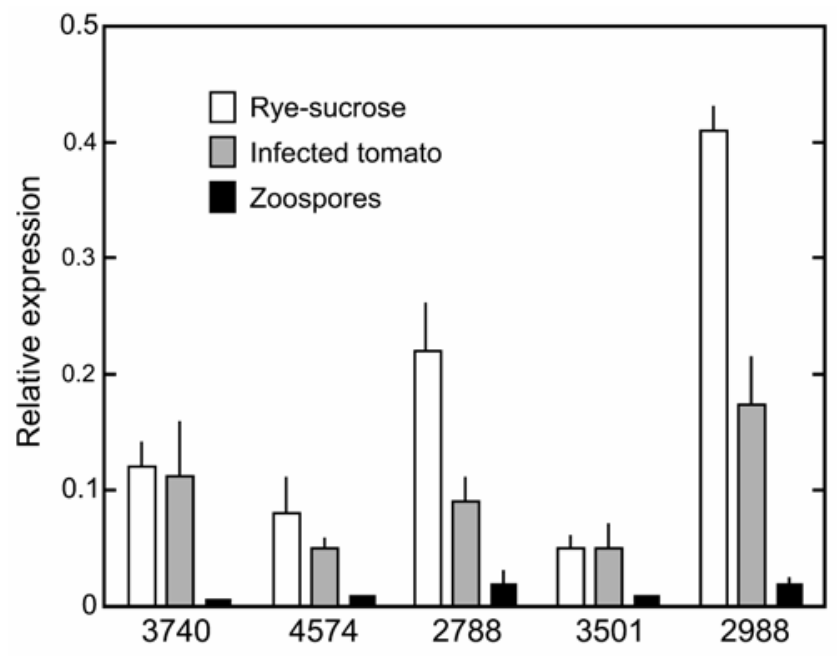

Fig. 6. Comparison of mRNA levels during in vitro and in planta culture. Quantitative reverse-transcription polymerase chain reaction assays were performed for genes Pi002788, Pi002988, Pi003501, Pi003740, and Pi004575 using RNA from 4-day nonsporulating hyphae from rye-sucrose media (open bars), 4-day infected tomato (gray), or swimming zoospores (black). Data are average of three determinations, normalized to ribosomal protein $\mathrm{S} 3 \mathrm{a}$. 
RNA was extracted by grinding in liquid nitrogen followed by use of the RNAEasy Plant Mini kit (Qiagen, Valencia, CA U.S.A.) according the manufacturer's instructions. RNA also was treated with RNase-free DNAse prior to use in hybridizations or RT-PCR assays.

\section{Microarrays and hybridizations.}

To obtain custom GeneChip arrays, Affymetrix (Santa Clara, CA, U.S.A.) was provided with 18,256 sequences combined from the $P$. infestans unigene set (Randall et al. 2005), other $P$. infestans sequences, and certain sequences from other Phytophthora spp. for probe design. Of these, 15,645 P. infestans sequences were judged by Affymetrix to meet their criteria for probe computation. These, plus 272 control sequences, were used by Affymetrix to design 13 sets of 25-nucleotide perfect and mismatch probes which were synthesized on GeneChips with 18-by-18- $\mu \mathrm{m}$ features. Sequences used for GeneChip design are deposited in the NCBI GEO archive.

Array experiments were performed as described by the Syngenta core facility (Zhu et al. 2001). Briefly, double-stranded cDNA was synthesized from $5 \mu \mathrm{g}$ of total RNA using an oligo $\mathrm{dT}_{(24)}$ primer containing a $\mathrm{T} 7$ promoter, and then biotinylated cRNA was produced using T7 RNA polymerase and fragmented. The probe array cartridge then was hybridized, washed, stained with streptavidin/phycoerythrin, and scanned. Spiking experiments indicated that the linear dynamic range of the GeneChips covered approximately 2.5 orders of magnitude, and that the sensitivity of detection was approximately $0.2 \mathrm{pM}$.

Each developmental stage was analyzed using two GeneChips, using RNA prepared by laboratories in North America and Europe. Each laboratory pooled RNA from two or three biological replicates. RNA was subjected to several forms of quality control prior to probe synthesis, including visualization by electrophoresis and calculation of the optical density at $260 / 280 \mathrm{~nm}$ ratio. As a control for RNA and cRNA quality, the ratio of expression signals from probe sets at the $5^{\prime}$ and $3^{\prime}$ ends of selected housekeeping genes were compared. For EF-1 $\alpha$ and ribosomal protein L3, for example, relative standard deviations between expression calls based on $5^{\prime}$ and $3^{\prime}$ probes were 10.7 and $10.4 \%$, respectively, among the 10 RNA samples examined in detail in this study.

\section{Microarray data analysis.}

Data was preprocessed using Affymetrix MAS 5.1 software. This included global normalization and scaling, and calculations of "present" and "absent" calls that indicate whether signal intensities were above background and specific to the targeted gene. Datasets then were stripped of genes from species other than $P$. infestans and reanalyzed using GeneSpring GX (Agilent Technologies, Santa Clara, CA, U.S.A.) after additional background correction, normalization to the 50th percentile, and per-gene normalization.

Differentially expressed genes were identified by filtering for fold changes, using replicates to set a $P<0.05$ threshold (based on $t$ test) and ensuring that "present" flags were called for each sample in which genes were considered to be expressed. Hierarchical clustering was performed using GeneSpring GX and EPCLUST, using the Pearson correlation method with average linkage. Iterative rounds of hierarchical clustering, K-means clustering, and visual inspection identified the 11 coregulated groups described above.

\section{Annotation of sequences.}

A two-round process was used to annotate genes represented on the GeneChips. Initially, unigene sequences were searched against the Swiss Protein database using BLASTX. Functions were extracted based on definition lines and domain annota- tions. Second, sequences falling into the expression clusters or having functions described above (transcription, DNA replication, and so on) were searched against whole-genome data; putative nonredundant full-length sequences then were compared with GenBank using BLASTX. This increased the number of informative matches by approximately $10 \%$. Automated gene models are still being refined; therefore, new BLAST matches obtained with the putative full-length sequences were examined manually to ensure accuracy. Manual analyses of 240 developmentally regulated unigenes indicated a rate of redundancy of approximately 6\%; in other words, cases where different parts of the same gene were represented by multiple unigenes.

\section{qRT-PCR.}

DNAse-treated RNA was reverse-transcribed using oligo-dT using first-strand synthesis kits from GE Healthcare (Little Chalfont, U.K.) or Invitrogen (Carlsbad, CA, U.S.A.) and subjected to PCR. Reactions were performed in an iCycler (BioRad, Richmond, CA, U.S.A.) or a 7700 Sequence Detection System (Applied Biosystems, Foster City, CA, U.S.A.) using the intercalation of SYBR Green as a fluorescence reporter and hot-start Taq polymerase (Applied Biosystems). Reactions were performed in triplicate using the following conditions: one cycle of $95^{\circ} \mathrm{C}$ for $8 \mathrm{~min}$ and 35 cycles of $95^{\circ} \mathrm{C}$ for $20 \mathrm{~s}$, $55^{\circ} \mathrm{C}$ for $20 \mathrm{~s}$, and $72^{\circ} \mathrm{C}$ for $30 \mathrm{~s}$. cDNA concentrations were normalized based on primers for the constitutively expressed gene encoding ribosomal protein S3a. Such normalization controls also were included in each run, and controls lacking reverse transcriptase also were performed. Fold changes were calculated from cycle threshold values assuming an efficiency in PCR of 1.9, which was the empirically determined average of 10 primer pairs. Genes chosen for analysis were picked from those having informative matches against non-oomycete proteins in GenBank, and for which raw signals on the GeneChips were above 100 .

\section{ACKNOWLEDGMENTS}

We thank Syngenta Corporation for providing the Affymetrix GeneChips and for funding some of the described analyses, T. Zhu for managing the processing of GeneChips, and T. Girke for assistance in database analyses. Other funding was provided to H. S. Judelson by the United States Department of Agriculture National Research Initiative Cooperative State Research Education and Extension Service and the Industry-University Cooperative Research Program of the University of California.

\section{LITERATURE CITED}

Ah Fong, A., and Judelson, H. S. 2003. Cell cycle regulator Cdc14 is expressed during sporulation but not hyphal growth in the fungus-like oomycete Phytophthora infestans. Mol. Microbiol. 50:487-494.

Avrova, A. O., Venter, E., Birch, P. R., and Whisson, S. C. 2003. Profiling and quantifying differential gene transcription in Phytophthora infestans prior to and during the early stages of potato infection. Fungal Genet. Biol. 40:4-14.

Avrova, A. O., Whisson, S. C., Pritchard, L., Venter, E., De Luca, S., Hein, I., and Birch, P. R. 2007. A novel non-protein-coding infection-specific gene family is clustered throughout the genome of Phytophthora infestans. Microbiology 153:747-759.

Bajorek, M., Finley, D., and Glickman, M. H. 2003. Proteasome disassembly and downregulation is correlated with viability during stationary phase. Curr. Biol. 13:1140-1144.

Baldauf, S. L., Roger, A. J., Wenk-Siefert, I., and Doolittle, W. F. 2000. A kingdom-level phylogeny of eukaryotes based on combined protein data. Science 290:972-977.

Bartnicki-Garcia, S., and Wang, M. C. 1983. Biochemical aspects of morphogenesis in Phytophthora. Pages 121-137 in: Phytophthora, its Biology, Taxonomy, Ecology, and Pathology. D. C. Erwin, S. BartnickiGarcia, and P. H. Tsao, eds. American Phytopathological Society Press, St. Paul, MN, U.S.A.

Bimpong, C. E. 1975. Changes in metabolic reserves activities during zoo- 
spore motility and cyst germination in Phytophthora palmivora. Can. J. Bot. 53:1411-1416.

Birch, P. R., Rehmany, A. P., Pritchard, L., Kamoun, S., and Beynon, J. L. 2006. Trafficking arms: Oomycete effectors enter host plant cells. Trends Microbiol. 14:8-11.

Blanco, F. A., and Judelson, H. S. 2005. A bZIP transcription factor from Phytophthora interacts with a protein kinase and is required for zoospore motility and plant infection. Mol. Microbiol. 56:638-648.

Clark, M. C., Melanson, D. L., and Page, O. T. 1978. Purine metabolism and differential inhibition of spore germination in Phytophthora infestans. Can. J. Microbiol. 24:1032-1038.

Cvitanich, C., and Judelson, H. S. 2003. A gene expressed during sexual and asexual sporulation in Phytophthora infestans is a member of the Puf family of translational regulators. Eukaryot. Cell 2:465-473.

Etienne, W., Meyer, M. H., Peppers, J., and Meyer, R. A., Jr. 2004. Comparison of mRNA gene expression by RT-PCR and DNA microarray. Biotechniques 36:618-620.

Fernandes, J., Brendel, V., Gai, X., Lal, S., Chandler, V. L., Elumalai, R. P., Galbraith, D. W., Pierson, E. A., and Walbot, V. 2002. Comparison of RNA expression profiles based on maize expressed sequence tag frequency analysis and micro-array hybridization. Plant Physiol. 128:896910.

Goernhardt, B., Rouhara, I., and Schmelzer, E. 2000. Cyst germination proteins of the potato pathogen Phytophthora infestans share homology with human mucins share homology with human mucins. Mol. PlantMicrobe Interact. 13:32-42.

Grenville-Briggs, L. J., Avrova, A. O., Bruce, C. R., Williams, A., Whisson, S. C., Birch, P. R., and van West, P. 2005. Elevated amino acid biosynthesis in Phytophthora infestans during appressorium formation and potato infection. Fungal Genet. Biol. 42:244-256.

Guldener, U., Seong, K. Y., Boddu, J., Cho, S., Trail, F., Xu, J. R., Adam, G., Mewes, H. W., Muehlbauer, G. J., and Kistler, H. C. 2006. Development of a Fusarium graminearum Affymetrix GeneChip for profiling fungal gene expression in vitro and in planta. Fungal Genet. Biol. 43:316-325.

Hardham, A. R. 2001. The cell biology behind Phytophthora pathogenicity. Aust. Plant Pathol. 30:91-98.

Hardham, A. R., and Hyde, G. J. 1997. Asexual sporulation in the oomycetes. Adv. Bot. Res. 24:353-398.

Hicke, L., and Dunn, R. 2003. Regulation of membrane protein transport by ubiquitin and ubiquitin-binding proteins. Annu. Rev. Cell Dev. Biol. 19:141-172.

Judelson, H. S., and Blanco, F. A. 2005. The spores of Phytophthora: Weapons of the plant destroyer. Nat. Microbiol. Rev. 3:47-58.

Judelson, H. S., and Roberts, S. 2002. Novel protein kinase induced during sporangial cleavage in the oomycete Phytophthora infestans. Eukaryot. Cell 1:687-695.

Judelson, H. S., and Tani, S. 2007. Transgene-induced silencing of the zoosporogenesis-specific PiNIFC gene cluster of Phytophthora infestans involves chromatin alterations. Eukaryot. Cell 6:1200-1209.

Kanneganti, T. D., Huitema, E., Cakir, C., and Kamoun, S. 2006. Synergistic interactions of the plant cell death pathways induced by Phytophthora infestans Nepl-like protein PiNPP1.1 and INF1 elicitin. Mol. Plant-Microbe Interact. 19:854-863.

Kim, K. S., and Judelson, H. S. 2003. Sporangia-specific gene expression in the oomyceteous phytopathogen Phytophthora infestans. Eukaryot. Cell. 2:1376-1385.

Krasinska, L., de Bettignies, G., Fisher, D., Abrieu, A., Fesquet, D., and Morin, N. 2007. Regulation of multiple cell cycle events by Cdc14 homologues in vertebrates. Exp. Cell Res. 313:1225-1239.

Lagow, E., DeSouza, M. M., and Carson, D. D. 1999. Mammalian reproductive tract mucins. Hum. Reprod. Update 5:280-292.

Latijnhouwers, M., and Govers, F. 2003. A Phytophthora infestans G-Protein $\beta$ subunit is involved in sporangium formation. Eukaryot. Cell 2:971-977.

Latijnhouwers, M., Ligterink, W., Vleeshouwers, V. G. A. A., van West, P., and Govers, F. 2004. A G-alpha subunit controls zoospore motility and virulence in the potato late blight pathogen Phytophthora infestans. Mol. Microbiol. 51:925-936.

Le Roch, K. G., Zhou, Y., Blair, P. L., Grainger, M., Moch, J. K., Haynes, J. D., De La Vega, P., Holder, A. A., Batalov, S., Carucci, D. J., and Winzeler, E. A. 2003. Discovery of gene function by expression profiling of the malaria parasite life cycle. Science 301:1503-1508.

Marshall, J. S., Ashton, A. R., Govers, F., and Hardham, A. R. 2001. Isolation and characterization of four genes encoding pyruvate, phosphate dikinase in the oomycete plant pathogen Phytophthora cinnamomi. Curr. Genet. 40:73-81.

Mattick, J. S. 2004. RNA regulation: A new genetics? Nat. Rev. Genet. 5:316-323.

Pazour, G. J., Agrin, N., Leszyk, J., and Witman, G. B. 2005. Proteomic analysis of a eukaryotic cilium. J. Cell Biol. 170:103-113.
Penington, C. J., Iser, J. R., Grant, B. R., and Gayler, K. R. 1989. Role of RNA and protein synthesis in stimulated germination of zoospores of the pathogenic fungus Phytophthora palmivora. Exp. Mycol. 13:158-168.

Prakob, W., and Judelson, H. S. 2007. Gene expression during oosporogenesis in heterothallic and homothallic Phytophthora. Fungal Genet. Biol. 44:726-739.

Qutob, D., Kamoun, S., and Gijzen, M., 2002. Expression of a Phytophthora sojae necrosis-inducing protein occurs during transition from biotrophy to necrotrophy. Plant J. 32:361-373.

Randall, T. A., Dwyer, R. A., Huitema, E., Beyer, K., Cvitanich, C., Kelkar, H., Ah Fong, A. M. V., Gates, K., Roberts, S., Yatzkan, E., Gaffney, T., Law, M., Testa, A., Torto, T., Zhang, M., Zheng, L., Mueller, E., Windass, J., Binder, A., Birch, P. R. J., Gisi, U., Govers, F., Gow, N. A. R., Mauch, F., van West, P., Waugh, M. E., Yu, J., Boller, T., Kamoun, S., Lam, S. T., and Judelson, H. S. 2005. Large-scale gene discovery in the oomycete Phytophthora infestans reveals likely components of phytopathogenicity shared with true fungi. Mol. Plant-Microbe Interact. 18:229-243.

Sachetto-Martins, G., Franco, L. O., and de Oliveira, D. E. 2000. Plant glycine-rich proteins: A family or just proteins with a common motif? Biochim. Biophys. Acta 1492:1-14.

Shan, W.-X., Marshall, J. S., and Hardham, A. R., 2004. Gene expression in germinated cysts of Phytophthora nicotianae. Mol. Plant Pathol. 5:317-330.

Smart, C. D., Fry, and W. E. 2001. Invasions by the late blight pathogen: Renewed sex and enhanced fitness. Biol. Invas. 3:235-243.

Solomon, P. S., Tan, K.-C., and Oliver, R. P. 2003. The nutrient supply of pathogenic fungi; a fertile field for study. Mol. Plant Pathol. 3:203-210.

Song, L., and Dentler, W. L. 2001. Flagellar protein dynamics in Chlamydomonas. J. Biol. Chem. 32:29754-29763.

Takano, Y., Choi, W., Mitchell, T. K., Okuno, T., and Dean, R. A. 2003. Large scale parallel analysis of gene expression during infection-related morphogenesis of Magnaporthe grisea. Mol. Plant Pathol. 4:337-346.

Tani, S., and Judelson, H. S. 2006. Activation of zoosporogenesis-specific genes in Phytophthora infestans involves a 7-nucleotide promoter motif and cold-induced membrane rigidity. Eukaryot. Cell 5:745-752.

Tani, S., Yatzkan, E., and Judelson, H. S. 2004. Multiple pathways regulate the induction of genes during zoosporogenesis in Phytophthora infestans. Mol. Plant-Microbe Interact. 17:330-337.

Todeschini, A. L., Morillon, A., Springer, M., and Lesage, P. 2005. Severe adenine starvation activates Ty1 transcription and retrotransposition in Saccharomyces cerevisiae. Mol. Cell Biol. 25:7459-7472.

Tsuruga, H., Yabuta, N., Hashizume, K., Ikeda, M., Endo, Y., and Nojima, H. 1997. Expression, nuclear localization and interactions of human MCM/P1 proteins. Biochem. Biophys. Res. Commun. 236:118-125.

Tyler, B. M., Tripathy, S., Zhang, X., Dehal, P., Jiang, R. H., Aerts, A., Arredondo, F. D., Baxter, L., Bensasson, D., Beynon, J. L., Chapman, J., Damasceno, C. M., Dorrance, A. E., Dou, D., Dickerman, A. W., Dubchak, I. L., Garbelotto, M., Gijzen, M., Gordon, S. G., Govers, F., Grunwald, N. J., Huang, W., Ivors, K. L., Jones, R. W., Kamoun, S., Krampis, K., Lamour, K. H., Lee, M. K., McDonald, W. H., Medina, M., Meijer, H. J., Nordberg, E. K., Maclean, D. J., Ospina-Giraldo, M. D., Morris, P. F., Phuntumart, V., Putnam, N. H., Rash, S., Rose, J. K., Sakihama, Y., Salamov, A. A., Savidor, A., Scheuring, C. F., Smith, B. M., Sobral, B. W., Terry, A., Torto-Alalibo, T. A., Win, J., Xu, Z., Zhang, H., Grigoriev, I. V., Rokhsar, D. S., and Boore, J. L.2006. Phytophthora genome sequences uncover evolutionary origins and mechanisms of pathogenesis. Science 313:1261-1266.

Warburton, A. J., and Deacon, J. W. 1998. Transmembrane $\mathrm{Ca}^{2+}$ fluxes associated with zoospore encystment and cyst germination by the phytopathogen Phytophthora parasitica. Fungal Genet. Biol. 25:54-62.

Whisson, S. C., Boevink, P. C., Moleleki, L., Avrova, A. O., Morales, J., Gilroy, E. M., Armstrong, M. R., Grouffaud, S., van West, P., Chapman, S., Hein, I., Toth, I. K., Pritchard, L., and Birch, P. R. J. 2007. A translocation signal for delivery of oomycete effector proteins inside host plant cells. Nature 450:115-118.

Yan, H. Z., and Liou, R. F. 2006. Selection of internal control genes for real-time quantitative RT-PCR assays in the oomycete plant pathogen Phytophthora parasitica. Fungal Genet. Biol. 43:430-438.

Zahiri, A. R., Babu, M. R., and Saville, B. J. 2005. Differential gene expression during teliospore germination in Ustilago maydis. Mol. Genet. Genomics 273:394-403.

Zhu, T., Budworth, P., Han, B., Brown, D., Chang, H.-S., Zou, G., and Wang, X. 2001. Toward elucidating the global gene expression patterns of developing Arabidopsis: Parallel analysis of 8300 genes by a high-density oligonucleotide probe array. Plant Physiol. Biochem. 39:221-242.

\section{AUTHOR-RECOMMENDED INTERNET RESOURCES}

Broad Institute of MIT and Harvard: www.broad.mit.edu 